\title{
Substrate determinants of Signal peptide peptidase-like 2a (SPPL2a)-mediated Intramembrane Proteolysis of the Invariant chain CD74
}

Short title: Substrate requirements of the intramembrane protease SPPL2a

\section{Susann Hüttl ${ }^{*}$, Felix Helfrich ${ }^{*}$, Torben Mentrup*, Sebastian Held ${ }^{*}$, Akio Fukumori†, Harald Steiner†‡, Paul Saftig ${ }^{\star}$, Regina Fluhrer†‡, and Bernd Schröder ${ }^{\star 1}$}

* Biochemical Institute, Christian Albrechts University of Kiel, Otto-Hahn-Platz 9, D-24118 Kiel, Germany

†DZNE - German Center for Neurodegenerative Diseases, Munich, Feodor-Lynen-Strasse 17, D-81377

Munich, Germany

‡ Biomedizinisches Centrum (BMC), Ludwig Maximilians University of Munich, Feodor-Lynen-Strasse 17, D-81377 Munich, Germany

${ }^{1}$ Correspondence should be addressed to:

Dr. Bernd Schröder, MD PhD

Biochemical Institute

Christian Albrechts University of Kiel

Otto-Hahn-Platz 9

D-24118 Kiel, Germany

Phone: +49-431-8802218; Fax: +49-431-8802238

E-mail: baschroeder@biochem.uni-kiel.de 


\section{ABBREVIATIONS}

The abbreviations used are: CLIP, class II-associated li chain peptide; ICD, intracellular domain; NTF, Nterminal fragment; SPPL, Signal-peptide-peptidase-like; TNF, Tumor Necrosis Factor $\alpha$.

\section{KEYWORDS}

Intramembrane proteolysis; Presenilin; Gamma-secretase; Signal-peptide-peptidase-like protease; CD74; Membrane protein degradation

\section{SUMMARY STATEMENT}

Intramembrane proteolysis of CD74 by SPPL2a is essential for B and dendritic cells. We show that CD74 is proteolyzed in the luminal third of the transmembrane segment and identify determinants within its transmembrane and luminal membrane-proximal domain facilitating this cleavage. 


\begin{abstract}
The presenilin-homologue Signal peptide peptidase-like 2a (SPPL2a) is an intramembrane protease of lysosomes/late endosomes which cleaves type II transmembrane proteins. We recently identified CD74, the invariant chain of the MHCII complex, as first in vivo validated substrate of this protease. In endosomal compartments, CD74 undergoes sequential proteolysis leading to the generation of a membrane-bound N-terminal fragment (NTF) that requires cleavage by SPPL2a for its turnover. In SPPL $2 a^{-/}$mice, this fragment accumulates in B cells and significantly disturbs their maturation and functionality. To date, the substrate requirements of the protease SPPL2a have not been investigated. Here, we systematically analyzed the molecular determinants of CD74 with regard to the intramembrane cleavage by SPPL2a. Using domain exchange experiments, we demonstrate that the intracellular domain of CD74 can be substituted without affecting cleavability by SPPL2a. Based on IP-MS analysis of the cleavage product, we report identification of the primary SPPL2a cleavage site between Y52 and F53 within the CD74 transmembrane segment. Furthermore, systematic alanine-scanning mutagenesis of the transmembrane and membrane-proximal parts of the CD74 NTF has been performed. We show that none of the analyzed determinants within the CD74 NTF including the residues flanking the primary cleavage site are absolutely essential for SPPL2a cleavage. Importantly, we found that alanine substitution of helixdestabilizing glycines within the transmembrane segment and distinct residues within the luminal membrane-proximal segment led to a reduced efficiency of SPPL2a-mediated processing. Therefore we propose that elements within the transmembrane segment and the luminal juxtamembrane domain facilitate intramembrane proteolysis of CD74 by SPPL2a.
\end{abstract}




\section{INTRODUCTION}

Intramembrane proteases of the GxGD type, which include the presenilins as catalytic subunits of the $\gamma$-secretase complex as well as signal peptide peptidase (SPP) and the related SPP-like (SPPL) proteases, have been implicated in diverse cellular processes [1,2]. Prominent functions of the $\gamma$-secretase complex include the mediation of Notch signaling and the generation of the $\mathrm{A} \beta$ fragment from the amyloid precursor protein which has been implicated in Alzheimer disease. In contrast, the in vivo functions of SPPL proteases, which exhibit selectivity for substrate proteins in type II orientation, are just beginning to emerge [2]. We and others recently showed that Signal peptide peptidase-like 2a (SPPL2a)-mediated intramembrane cleavage of CD74, the invariant chain of the MHCII complex, is an essential process in B cells [3-5]. This view is based on significantly disturbed development and functionality of B cells and dendritic cells in SPPL2a $a^{-/}$mice which is caused by the accumulation of an CD74 N-terminal fragment (NTF) in endosomal membranes that disturbs central signaling pathways [3,6]. Within the SPP/SPPL family, SPPL2a is the only member that is predominantly localized in lysosomes/late endosomes $[7,8]$. Consistently, the role of SPPL2a for the cleavage of CD74 cannot be compensated by any other member of this protease family in vivo, despite the general capability of SPPL2b to cleave CD74 upon coexpression of substrate and protease in vitro [9]. Further reported substrates of SPPL2a include TNF $\alpha$ $[8,10]$, the Fas ligand [11] and the proteins ITM2B [12] and TMEM106B [13]. However, their identification was achieved in cell-based experiments and the physiological relevance of these cleavage events at the endogenous level in vivo remains to be determined.

CD74 is primarily known for its prominent role in antigen presentation by facilitating assembly and subcellular targeting of MHCII complexes. During this process, the CLIP segment (class II-associated li chain peptide) of CD74 prevents premature binding of peptides to the MHCII complex within the secretory pathway. After delivery to endosomal compartments, different luminal proteases sequentially process the CD74 ectodomain releasing MHCII. Several endosomal proteases have been reported to be involved in this process including the asparaginyl-endopeptidase legumain and the cathepsins $\mathrm{S}, \mathrm{L}$ and $\mathrm{F}$ $[14,15]$. However, the individual contribution of these and possibly other proteases to this process differs in a cell-type specific manner. Finally, a membrane-bound CD74 NTF remains that cannot be further processed by luminal proteases and depends on the intramembrane cleavage by SPPL2a to be cleared from the membrane. Thereby, the CD74 intracellular domain (ICD) is liberated as cleavage product into the cytosol which may have regulatory functions [16,17]. In parallel, a second fragment (C-peptide) is released towards the extracellular/luminal face of the membrane.

Based on the distinct immunological phenotype of SPPL $2 a^{-/}$mice [3-6], inhibition of this enzyme has been proposed as a therapeutic strategy to deplete and/or inhibit B and dendritic cells. Currently, the molecular determinants within substrate proteins that are required for the intramembrane cleavage by SPPL2a are unknown, since no structure-function analysis for this protease has been performed. Here, we systematically investigate the substrate determinants of CD74 required for cleavage by SPPL2a using alanine-scanning mutagenesis and domain exchange experiments. Our results suggest that the intracellular domain of CD74 does not contain essential determinants that are required for the cleavability of the protein. However, we demonstrate that the luminal juxtamembrane segment of CD74 and helixdestabilizing glycines within the transmembrane segment facilitate processing by SPPL2a. Furthermore, we report mass-spectrometric identification of the primary SPPL2a cleavage site within the CD74 NTF. 


\section{EXPERIMENTAL}

\section{Reagents}

The SPP/SPPL inhibitor (Z-LL) 2 ketone (Calbiochem) was used at $20 \mu \mathrm{M}$. ER retention of newly synthesized proteins was induced with brefeldin A (Enzo) at $1 \mu \mathrm{g} / \mathrm{ml}$. Both compounds were dissolved in DMSO.

\section{Molecular cloning and cDNA constructs}

All CD74 expression constructs were generated in pcDNA3.1/Hygro ${ }^{+}$(Invitrogen) based on the cDNA of the $\mathrm{p} 31$ isoform of murine $\mathrm{CD} 74$ that has been described before $[3,9]$. In most of the employed constructs, the CD74 open reading frame was fused at its 3' end to the coding sequence of an HA epitope (mCD74HA). In some cases, constructs comprising an N-terminal HA and a C-terminal V5 tag (HA-mCD74-V5) were utilized for means of detectability. Mutations were introduced by overlap-extension PCR to produce different alanine substitution variants of CD74. Chimeric constructs in which the coding sequence of amino acids 2-29 of murine CD74 was replaced as depicted in Figure 3A were used to analyze the impact of CD74 ICD on cleavage by SPPL2a. As a substitute, coding sequences of amino acids 2-47 from human transmembrane protein 192 (TMEM192) [18] or residues 2-52 from human Disrupted in renal carcinoma 2 (DIRC2) [19] were used. Thus, amino acids 30-215 of the murine CD74 p31 isoform were preserved in the TMEM192 $2_{\mathrm{NT}}-\mathrm{mCD} 74$ and DIRC2 $2_{\mathrm{NT}}-\mathrm{mCD} 74$ chimeric proteins. The respective cDNA fragments were amplified and fused by overlap-extension PCR. Coding sequences for an HA and a V5 epitope were added to the $5^{\prime}$ and the 3 '-end of the open reading frame, respectively, prior to insertion into pcDNA3.1/Hygro ${ }^{+}$ (Invitrogen) via BamHI and XhoI restriction sites. For cleavage site determination, coding sequences of the CD74 NTF comprising amino acids 2-82 of murine CD74 p31, an N-terminal V5 epitope (MGKPIPNPLLGLDST) and a C-terminal FLAG epitope (DYKDDDDK) were subcloned into pcDNA 3.1/ Hygro $^{+}$using BamHI and XhoI restriction sites. At the very C-terminus an alanine-proline motif was added to prevent degradation of liberated fragments by exopeptidases (Figure 2). Expression plasmids of murine SPPL2a and its inactive D416A mutant with a C-terminal Myc tag have been described before $[3,7]$. The murine cathepsin S (CTSS) ORF was amplified from murine splenic cDNA and inserted into pcDNA3.1/Hygro ${ }^{+}$via introduced BamHI and $\mathrm{XhoI}$ restriction sites.

\section{Cell lines and transfection}

The human melanoma cell line MelJuso was cultivated in RPMI 1640 with L-glutamine (Sigma) supplemented with $10 \% \mathrm{v} / \mathrm{v}$ fetal bovine serum (Biochrom), $100 \mathrm{U} / \mathrm{ml}$ penicillin (Sigma) and $100 \mu \mathrm{g} / \mathrm{ml}$ streptomycin (Sigma). HeLa (DSMZ) and HEK293 EBNA (Invitrogen) cells were grown in Dulbecco's modified Eagle's medium with glutamine or Glutamax (Invitrogen), respectively, with the same supplements. All cell lines were cultured at $37^{\circ} \mathrm{C}$ in a humidified $95 \%$ air $/ 5 \% \mathrm{CO}_{2}$ atmosphere. HEK293TR cells stably expressing human SPPL2a have been described before [20]. To induce expression of SPPL2a, cells were incubated with $1 \mu \mathrm{g} / \mathrm{ml}$ doxycycline (BD Biosciences) added to the cell culture medium for at least $48 \mathrm{~h}$. For transfections and inhibitor experiments, cells were seeded the preceding day. Transient transfections of MelJuso, HeLa and HEK293 cells were performed with Turbofect (Thermo Scientific) or Lipofectamin 2000 (Invitrogen) according to the manufacturer's recommendations. When co-transfections of substrate and protease(s) were performed, the total amount of transfected DNA was kept constant and balanced, where necessary, using empty pcDNA3.1/Hygro ${ }^{+}$. To reduce cytotoxicity, the culture medium was replaced $6 \mathrm{~h}$ post-transfection. Knockdown of endogenous SPPL2a in MelJuso cells was performed with a pool of four gene-specific siRNAs (SMARTpool: GUUGUUGCCUGGAGACGUA, GGAGUGGACUAGUUGAAUU, CCUCAUGCCUGUUUCAAUA, GGUAACAGCUAUCAGAUGA) as well as of control siRNAs. These were purchased from Thermo 
Scientific and transfected using INTERFERin (Polyplus transfection) following the supplier's recommendations at a final concentration of $50 \mathrm{nM}$.

\begin{abstract}
Western blotting
Total cell extracts were prepared as described before [18]. In brief, cells were harvested and lysed in $50 \mathrm{mM}$ Tris- $\mathrm{HCl}(\mathrm{pH} 7.4), 150 \mathrm{mM} \mathrm{NaCl}, 1.0 \%(\mathrm{w} / \mathrm{v})$ Triton X-100, $0.1 \%(\mathrm{w} / \mathrm{v})$ SDS and $4 \mathrm{mM}$ EDTA supplemented with protease inhibitors [18]. Protein concentrations were determined applying a bicinchoninic acid (BCA) protein assay (Thermo Scientific). Lysates were subjected to electrophoretic separation by SDS-PAGE. Depending on the protein to be detected, either Tris-tricine [21] or Tris-glycine [22] buffer systems were employed. Subsequently, proteins were transferred to nitrocellulose for immunodetection [18]. For detection of murine CD74, we employed the established rat monoclonal antibody In-1 [23,24] which recognizes a cytoplasmic epitope of murine CD74 (BD Biosciences). To reveal endogenous human CD74 in the MelJuso cells, the monoclonal PIN1 antibody (StressMarq Biosciences) was utilized. Generation of rabbit polyclonal antibodies against murine SPPL2a [7] has been described previously. Human SPPL2a was visualized with a rabbit polyclonal antiserum against a internal epitope of this protein (CENLKAVTTEDREMRK, residues 196-210) which was affinity-purified against the immobilized peptide (Pineda Antikörper-Service). In addition, the 3F10 rat monoclonal antibody against the HA epitope was used (Roche) to detect epitope tagged variants of CD74. To assess protein loading, we used rabbit polyclonal antibodies against $\beta$-Actin (Sigma-Aldrich), GAPDH (Sigma-Aldrich) or elongation factor 2 (EEF2; Abcam) or a mouse monoclonal antibody against $\beta$-tubulin (E7, Developmental Studies Hybridoma Bank). HRP-coupled secondary antibodies for chemiluminescent detection were purchased from Dianova. Densitometric quantification of band intensities was performed with ImageJ (National Institutes of Health).
\end{abstract}

\title{
Indirect immunofluorescence
}

Cells seeded on glass coverslips were fixed with $4 \%(\mathrm{w} / \mathrm{v})$ paraformaldehyde in phosphate-buffered saline (PBS) for $20 \mathrm{~min}$ at room temperature prior to immunocytochemical staining according to the procedure described in [18]. To prevent unspecific antibody binding, samples were incubated for a minimum of $2 \mathrm{~h}$ in blocking solution $(10 \%(\mathrm{v} / \mathrm{v})$ fetal bovine serum in PBS). The expressed murine CD74 was visualized using the monoclonal In-1 antibody diluted in blocking solution. If detection with In-1 was not possible due to the absence of the epitope (18-23A mutant, TMEM192 $2_{\mathrm{NT}}-\mathrm{mCD} 74$ and DIRC2 $2_{\mathrm{NT}}-\mathrm{mCD} 74$ chimeras), constructs with an N-terminal HA epitope were employed and visualized using the 3F10 antibody. Expression of co-transfected murine SPPL2a was detected via a C-terminal Myc epitope using the mouse monoclonal 9B11 antibody (Cell signaling). A polyclonal antibody against the ER marker protein PDI (protein disulfide isomerase) was obtained from Abcam and secondary antibodies conjugated to Alexa 488 and -594 were from Life Technologies. Coverslips were embedded in Mowiol containing DABCO (1,4-diazobicyclo-(2.2.2) octane) and DAPI (4-,6-diamidino-2-phenylindole). A FV1000 confocal laser scanning microscope (Olympus) was used to acquire pictures from optical sections. Images were processed with Olympus Fluoview Software (3.0a) and Adobe Photoshop.

\section{Cleavage site determination}

Conditioned media of cells indicated in the respective figure were collected after $18 \mathrm{~h}$ and CD74 NTF cleavage products were purified by immunoprecipitation using anti-Flag M2-conjugated agarose beads. Following agitation at $4{ }^{\circ} \mathrm{C}$ overnight, beads were washed three times with IP/MS buffer $(0.1 \% \mathrm{~N}$ octylglucoside, $10 \mathrm{mM}$ Tris-HCl, $\mathrm{pH} 8.0,5 \mathrm{mM}$ EDTA, $140 \mathrm{mM} \mathrm{NaCl}$ ) and three times with sterile water. Immunoprecipitated peptides were eluted with trifluoroacetic acid/acetonitrile/water $(1: 20: 20)$ saturated 
with $\alpha$-cyano-4-hydroxy cinnamic acid. The dissolved samples were dried on a stainless plate and subjected to MALDI-TOF MS analysis using a Voyager DE STR (Applied Biosystems). 


\section{RESULTS}

\section{Proteolytic processing of CD74 in the melanoma cell line MelJuso}

We aimed to gain insight into the structural determinants within murine CD74 that are required for the intramembrane cleavage by SPPL2a. Therefore, we employed the human melanoma cell line MelJuso as model system due to its good transfectability and expression of components of the MHCII antigen presentation machinery [25]. The CD74 topology and the current model of the sequential processing of this protein are depicted in Figure 1A. Since we aimed to determine the cleavage of murine CD74, we examined the subcellular distribution of heterologously expressed murine CD74 and SPPL2a in MelJuso cells. Upon co-expression, both proteins showed a partially overlapping distribution in immunofluorescence analysis which is a prerequisite for proteolysis (Figure 1B). In accordance with previous studies, CD74-positive vesicles frequently appeared to be enlarged as compared to endosomal compartments in non-transfected MelJuso cells [26-29]. We also confirmed that MelJuso cells endogenously express CD74 and SPPL2a (Figure 1C). Upon siRNA-mediated knockdown of SPPL2a, a prominent N-terminal fragment of the endogenous CD74 was found to be stabilized (Figure 1C) which was accompanied by a second significantly less intense fragment. This indicates that in these cells intramembrane proteolysis of CD74 by SPPL2a takes place under endogenous conditions and that they represent an appropriate experimental system for the devised study.

We expressed murine CD74 in MelJuso cells and performed Western blot analysis using an antibody against an N-terminal epitope of murine CD74 (Figure 1D). This revealed the CD74 full-length protein (FL) and several C-terminally truncated N-terminal fragments (NTF) as reported before $[3,9]$. These different NTFs exhibited apparent molecular weights between 10 and $17 \mathrm{kDa}$ and appeared as two blocks of bands that will be referred to as NTF1 and NTF2 in the following (Figure 1D). Each of these NTF blocks was constituted by at least two individual, though difficult-to-resolve bands which exhibited very similar electrophoretic migration. Treatment of transfected cells with brefeldin A, which blocks trafficking within the early secretory pathway, prevented generation of any NTFs and stabilized the fulllength protein (Figure 1D). This demonstrates that the brefeldin A-induced ER retention (Figure 1E) completely prevents proteolytic degradation of CD74 in agreement with the known role of endosomal proteases in this process $[14,15,30]$. Co-expression of catalytically active SPPL2a, but not of the catalytically inactive D416A (SPPL2a-D/A) mutant, diminished the smaller NTF2 (Figure 1F). The antibodies utilized for the detection of the expressed murine CD74 (mCD74) and SPPL2a (mSPPL2a) did not cross-react with the corresponding human proteins as revealed by the absence of immunoreactive bands in cells transfected with empty vector (Figure 1F). In addition to NTF2, longer CD74 N-terminal fragments (NTF1) were also found to be modulated to a significant degree by co-expressed SPPL2a. However, often the depletion was less complete and in general more variable than that of NTF2. This observation may suggest an inverse correlation between NTF fragment size and SPPL2a cleavage efficiency in this system. Such a less efficient cleavage of the longer CD74 NTF1 could be due to the requirement of a short substrate ectodomain by SPPL2 proteases as it was described previously for SPPL2b [12]. In agreement with previous reports [3,9], the abundance of the CD74 full-length protein was not altered by protease co-expression. We also assessed if the murine CD74 can undergo processing by the endogenously present human SPPL2a. This was confirmed by a significant accumulation of the CD74 NTF2 in transfected MelJuso cells upon knockdown of endogenous SPPL2a (Fig. 1G), whereas NTF1 was not modulated. This supports the view that NTF2 is the primary SPPL2a substrate. Altogether, the findings confirm suitability of the selected cell line to assess cleavage of heterologously expressed CD74 by co-expressed SPPL2a. However, delivery of CD74 to endosomal compartments is a prerequisite for processing of the luminal domain and generation of the NTFs which are the actual substrates of SPPL2a.

\section{Determination of the SPPL2a-cleavage site in the CD74 NTF}

In order to determine the primary cleavage site within the CD74 transmembrane segment, we directly expressed a CD74 NTF comprising amino acids 2-82 in HEK293 cells which has been reported to be the 
site where the CLIP segment is proteolytically separated from the residual membrane-bound NTF prior to the intramembrane cleavage $[30,31]$. By this means, we aimed to avoid heterogeneity resulting from processing of the luminal domain and to enable direct cleavage of the NTF by SPPL2a already within the biosynthetic pathway. Therefore, the C-peptides originating from the intramembrane cleavage could be recovered from the cell culture medium by immunoprecipitation using antibodies against the $\mathrm{C}$-terminal FLAG epitope (Figure 2A). Mass-spectrometric analysis identified several proteolytic fragments specific to CD74 (Figure 2B). Among these, particularly the intensity of the peak with a determined mass of 4755 $\mathrm{Da}$ (Figure 2B, black arrowhead) increased upon SPPL2a co-expression, suggesting that cleavage between Y52 and F53 of murine CD74 reflects the major SPPL2a cleavage site. Concurrent application of the SPP/SPPL inhibitor (Z-LL) 2 -ketone significantly reduced the intensity not only of the described but also of several other peaks (Figure 2B) indicating that these peptides also result from an SPP/SPPL mediated intramembrane cleavage of CD74. We compared the mass of the observed peaks to the peptide masses that are expected based on the CD74 protein sequence (Figure 2C). This revealed that the detected peptides match protein sequences N-terminally starting between T49 and Y55. Similar cleavage patterns, depicting one major cleavage site and several minor flanking cleavage sites have also been observed for other members of the SPP/SPPL family [10,32]. Therefore, we conclude that the cleavage of CD74 by SPPL2a that leads to the liberation of the C-peptide towards the extracellular/luminal side of the membrane takes place between T49 and Y 55 of murine CD74 with a major cleavage site between Y 52 and F53.

\section{Exchange of the CD74 cytosolic domain does not impair cleavage by SPPL2a}

We aimed to systematically determine the importance of different parts of the CD74 NTF for recognition and cleavage by SPPL2a. In order to assess the role of the ICD segment (aa 2-29), we created chimeric proteins in which this part of the murine CD74 p31 isoform was replaced by cytosolic tails of two unrelated lysosomal proteins (Figure 3A), while the transmembrane segment and luminal domain of murine CD74 were preserved. Since endosomal targeting of CD74 depends on two dileucine based motifs within its ICD (LI7/8, IL16/17, Figure 3A) [33], we selected the N-terminal cytosolic segments of the lysosomal multipass membrane proteins Disrupted in renal carcinoma 2 (DIRC2) and Transmembrane protein 192 (TMEM192) as substitutes that also harbor functional dileucine-based sorting motifs [19,34]. For means of detection, an N-terminal HA and a C-terminal V5 epitope were added (Figure 3A). In MelJuso cells, the expression levels observed after transient transfection of these chimeric DIRC2 $2_{\mathrm{NT}}-$ mCD74 and TMEM192 ${ }_{\mathrm{NT}}-\mathrm{mCD} 74$ proteins were rather low in comparison to wild type CD74 (data not shown). We assumed that this could reflect an interference between the chimeric proteins and the endogenously present CD74 and MHCII in the MelJuso cells and, therefore, performed the analysis of these chimeric proteins in HeLa cells. Targeting of the DIRC2 $2_{\mathrm{NT}}-\mathrm{CD} 74$ and TMEM192 $2_{\mathrm{NT}} \mathrm{CD} 74$ chimeric proteins to endosomal compartments and co-localization with co-expressed SPPL2a was comparable to wild type CD74 (Figure 3B) as was proteolytic processing of the luminal domain (Figure 3C). Most importantly, SPPL2a-mediated depletion of the NTF2 was observed irrespective of being derived from the CD74 wild type protein or the chimeric protein with the exchanged ICD. This indicates that the cytosolic part of CD74 does not comprise any sequence determinants that are absolutely required for the intramembrane cleavage by SPPL2a. However, we cannot exclude that the cytoplasmic domains of DIRC2 and TMEM192 share unknown structural features with the CD74 ICD which promote cleavage of the chimeric proteins.

\section{Systematic alanine-scanning mutagenesis of the CD74 NTF}

Next we investigated the relevance of the transmembrane and luminal membrane-proximal segment of CD74 for proteolysis by SPPL2a. Therefore, we pursued generation of additional chimeric proteins by replacing these parts with those from other type II or multi-pass transmembrane proteins. However, in the vast majority these chimeric proteins were not properly expressed and did not reach endosomal 
compartments, thus, precluding any conclusive analysis (not shown). Therefore, we performed systematic alanine-scanning mutagenesis up to amino acid 82 of CD74 (Figure 4A). Based on the essential role of lysosomal/endosomal targeting motifs at positions $7 / 8$ and $16 / 17$ and the results achieved with chimeric model proteins (Figure 3) arguing against a major role of this protein part, residues 2-17 were not included into this mutagenesis study. All alanine-scanning expression constructs were devoid of any $\mathrm{N}$-terminal epitope tag in order to mimic the physiological situation as closely as possible. We analyzed the subcellular targeting of all CD74 mutants in transfected MelJuso cells by indirect immunofluorescence to assess their co-localization with co-expressed SPPL2a as a prerequisite for proteolysis (data not shown). In this analysis, we failed to detect expression of the 18-23A and 52-54A CD74-HA constructs. However, all remaining CD74 alanine mutants listed in Figure 4A, except for 38-40A and 42-44A, exhibited overlapping distributions with SPPL2a like the wild type protein.

Proteolytic processing of the 30A, 32-37A, 46-50A, 61-66A, 67-72A, 73-78A and 79-82A mutant proteins was found to occur in a comparable way to wild type CD74 (Figure 4B). The respective mutant NTFs (primarily NTF2) were efficiently depleted by co-expressed SPPL2a. This already indicates that significant parts of the transmembrane segment and the luminal membrane-proximal domain of CD74 are not absolutely required for the intramembrane cleavage by SPPL2a and can be exchanged without losing cleavability. Interestingly, electrophoretic migration of some of the mutant NTFs was slower than of those from wild type CD74 although the general band pattern was preserved. In this initial analysis, six mutants appeared to behave differently from wild type CD74 in Western blot analysis: This includes the 18-23A mutant which was not detected at all in this setup as well as the 38-40A, 42-44A, 52-54A mutants which exhibited very low overall expression levels. In contrast, the full-length form of 24-29A mutant CD74 was present, but only very little NTF derived from this mutant was observed under these conditions. Furthermore, the SPPL2a-mediated depletion of the NTF2 from the 55-60A mutant CD74 repeatedly appeared to be less complete than for the wild type protein. The underlying cause for these described differences will be investigated in detail in the following.

As mentioned, the 18-23A CD74-HA mutant protein completely failed to be detected with the In1 antibody (Figure 4B). However, expression could be confirmed by detection of the C-terminally fused HA tag that was present in all alanine-scanning mutants (Figure 4A). This indicates that residues 18-23 are an essential part of the epitope recognized of the In- 1 antibody. Therefore, we reanalyzed this CD74 mutant using an N-terminaly HA tagged CD74 mutant construct (Figure 4C). Endosomal targeting of this mutant was similar to the corresponding wild type CD74 protein (data not shown). In line with the results from the chimeric proteins (Figure 3), substitution of amino acids 18-23 did not impair SPPL2a-mediated proteolysis since depletion of NTF2 occurred in a similar manner as for wild type CD74.

\section{Palmitoylation influences NTF levels, but is no prerequisite for intramembrane proteolysis}

As depicted in Figure 4B, we observed consistently low steady-state NTF levels upon expression of the 24-29A CD74 mutant. Subcellular targeting of the 24-29A CD74 as assessed by immunofluorescence was comparable to that of wild type CD74 (data not shown). This excludes mis-targeting to the plasma membrane or ER retention which could account for a reduced production of the NTF. However, it has been reported that CD74 undergoes palmitoylation on cysteine residue C27 [35-37]. Absence of this modification could well account for decreased stability of the 24-29A CD74 mutant. In order to analyze this in more detail, we created a C27A CD74 mutant and expressed it in MelJuso cells. As expected, exchange of the single cysteine residue did not affect subcellular targeting of CD74 in MelJuso cells (Figure 5A). Western blot analysis revealed a processing pattern of the C27A mutant that was characterized by considerably reduced steady-state NTF levels in comparison to the wild type protein, reflecting the initial observations from the 24-29A mutant. Altogether, this suggests that the failure to become lipidated accounts to a major degree for the low NTF levels of both mutants. Despite its lower abundance, the C27A NTF could be visualized on longer Western blot exposures. There, we observed that co-expressed SPPL2a was capable of further reducing the abundance of the C27A NTF. Thus, it seems that non-palmitoylated CD74 can undergo intramembrane proteolysis and that acylation is in principle 
dispensable for this process even though this posttranslational modifications seems to modulate CD74 processing in general.

\section{Residues covering the primary cleavage site of SPPL2a are not essential for proteolysis}

Three mutants with substitution of the amino acid triplets 38-40, $42-44$ and 52-54 were found to exhibit very low expression levels in transiently transfected MelJuso cells as compared to wild type CD74 (Figure 4B). All of these substituted residues are part of the transmembrane segment. Amino acids 52-54 appeared to be of particular interest since this stretch covers the primary cleavage site of SPPL2a (Figure 2) that leads to the liberation of the C-peptide. No NTFs derived from the 38-40A, 42-44A and 52-54A mutant proteins were detected. As mentioned above, immunofluorescence analysis failed to reveal co-localization between the 38-40A and 42-44A CD74 mutants and co-expressed SPPL2a (data not shown). Instead both mutant proteins were detected in the endoplasmic reticulum to major degree based on their overlapping distribution with PDI (data not shown). Since the 52-54A mutant was not reliably detected by immunofluorescence due to its low expression level, the subcellular localization of this protein could not be determined. Altogether this suggests that replacing amino acids 38-40, 42-44 and 52-54 substantially interferes with folding and/or membrane insertion of CD74. However, in the absence of endosomal targeting and NTF generation, any potential effect of these mutations on the cleavage by SPPL2a could not be assessed.

Therefore, we sought to scrutinize the role of these residues further by investigating mutants with exchange of individual amino acids of the respective triplets. All nine CD74 mutant proteins with single alanine substitutions (V38A, L39A, V40A, L42A, L43A, L44A, Y52A, F53A, L54A) were correctly targeted and showed co-localization with co-expressed SPPL2a (Figure 6A). In agreement with this, NTFs derived from all of these mutant proteins were detected confirming proteolytic processing of the luminal domain similar to that of the wild type protein (Figure 6B-D). Upon co-expression of SPPL2a, all nine single mutants were processed by SPPL2a indicating that none of these amino acid residues represents an essential determinant for proteolysis by SPPL2a. Even the tyrosine and phenylalanine residues in the P1 and P1' position could be replaced with alanine residues without abolishing cleavability of CD74.

\section{Helix-destabilizing glycine residues are not essential, but facilitate the intramembrane cleavage of CD74}

In a previous study, helix-destabilizing glycine residues within the transmembrane domain have been described to be of critical importance for processing of the ITM2B (Bri2) protein by the SPPL2ahomologue SPPL2b [38]. Based on this, we generated a CD74 expression construct termed GallA where all three glycines within the transmembrane segment (G30, G35 and G46) were substituted by alanines (Figure 7A). CD74 GallA co-localized with SPPL2a similar to wild type CD74 (Figure 7B) allowing to assess its cleavage by SPPL2a. Co-expressed SPPL2a clearly reduced the NTF2 derived from the GallA mutant indicating that the three glycine residues are not essential for the cleavage (Figure 7C). However, we noticed repeatedly that minor amounts of the CD74 GallA NTF2 remained detectable upon SPPL2a co-expression. When we quantified the residual NTF2, which was normalized to the abundance of the fulllength protein, the presence of SPPL2a reduced the wild type NTF2 to less than $10 \%$ of the level detected in cells without protease overexpression (Figure 7D). In comparison, the depletion of the GallA NTF2 appeared to be less complete reflecting the described increased amounts of residual NTF2 as depicted in Fig 7B. This could indicate that the efficiency of SPPL2a-mediated processing of CD74 is slightly reduced by the GallA mutation, although cleavability in general is not abolished and the effect is subtle. We attempted to further substantiate this observation by increasing the supply of the CD74 NTF as the actual SPPL2a substrate. Cathepsin S (CTSS) has been implicated in processing CD74 at different sites, however, especially in cleaving off the CLIP segment (Figure 1A) [39].Therefore, we tried to enhance processing of CD74 by co-expression of CTSS (Figure 7E). In cells transfected with wild type CD74, this procedure affected NTF1 that appeared as a single prominent band upon CTSS expression. Furthermore, 
we observed a slight increase of the NTF2 steady-state levels which is the one preferably cleaved by SPPL2a. However, also in CTSS expressing cells co-expression of SPPL2a led to an almost complete depletion of NTF2 from wild type CD74. In contrast, small amounts of residual NTF2 were detected when the GallA mutant was analyzed, thus corroborating the view that the cleavage efficiency of the latter is subtly impaired. This data support the conclusion that helix-destabilizing glycines within the transmembrane region of $\mathrm{CD} 74$ are not essential for, but may facilitate intramembrane proteolysis by SPPL2a.

\section{Determinants within the luminal membrane-proximal segment promote CD74 processing}

As described above, the NTF2 derived from CD74 with alanine substitution of residues 55-60 (55-60A) appeared to be less efficiently removed by co-expressed SPPL2a (Figure 4B). Though co-expressed SPPL2a reduced the amount of the NTF2 derived from the 55-60A mutant to some extent, we consistently observed higher residual amounts of the 55-60A CD74 NTF2 in SPPL2a-expressing cells as compared to the wild type NTF2 (Figure 8A). SPPL2a co-expression reduced the 55-60A NTF2 only to about $40 \%$ of the level without SPPL2a transfection in contrast to the wild type NTF2 from which less than $10 \%$ remained (Figure $8 \mathrm{~B}$ ). In order to promote the generation of NTF2 and thereby increase the substrate supply, we performed co-expression with CTSS (Figure 8A). This further enhanced the difference between the wild type CD74 and the 55-60A mutant NTF2 substantiating that the 55-60A NTF2 is incompletely processed by SPPL2a under these experimental conditions.

Having identified a role of the 55-60 stretch in facilitating proteolysis by SPPL2a, we intended to further narrow the amino acid residues responsible for this effect. Therefore, we generated three additional mutants where only two consecutive residues were substituted by alanines (YQ55/56AA, QQ57/58AA, GR59/60AA). Like the 55-60A mutant, these three mutants exhibited an overlapping subcellular localization with co-expressed SPPL2a (Figure 8C). We transfected these constructs into MelJuso cells together with SPPL2a (Figure 8D,E) or SPPL2a and CTSS (Figure 8F). Neither mutation of amino acids $55 / 56$ nor of amino acids 57/58 affected processing of CD74 NTF2 by SPPL2a. Importantly, the 59/60A mutant recapitulated the findings for the 55-60A mutant (Figure 8A, B). Processing of the 59/60 NTF2, though not generally abolished, was found to be less efficient than for the wild type protein which became evident by significantly higher residual NTF2 levels upon SPPL2a overexpression (Figure 8E). Interestingly, the electrophoretic migration of the 59/60A NTF2 appeared to be slightly altered as compared to wild type CD74 possibly reflecting the substitution of a positively charged arginine residue by alanine.

Having identified the membrane-proximal residues 59/60 and the helix-destabilizing glycines in the transmembrane segment as facilitators of SPPL2a cleavage we wanted to know if their impact on CD74 proteolysis is additive. Therefore, we combined the respective mutants in one construct (GallAGR59/60AA) and compared cleavability of this combination mutant to the GR59/60AA single mutant and wild type CD74 (Figure 9A,B). Using indirect immunofluorescence, we confirmed correct targeting of the GallA-GR59/60AA mutant and demonstrated an overlapping subcellular distribution with co-expressed SPPL2a (Figure 9C). In order to be able to detect subtle changes in cleavage efficiency, MelJuso cells transiently expressing the three CD74 variants were co-transfected with increasing amounts of active SPPL2a (Figure 9A). Most importantly, also the combination of the GallA and the GR69/60AA mutations did not render CD74 uncleavable. The mutant NTF2 was depleted to a certain degree by co-expressed SPPL2a. However, as described before for the GR59/60AA mutant (Figure 8) this depletion was incomplete and $\sim 20 \%$ residual NTF2 remained detectable even at increasing SPPL2a levels. Upon high SPPL2a protease levels, the GallA-GR59/60AA combination mutant mainly reflected the behavior of the GR59/60AA (Figure 9B). However, we observed repeatedly a slight tendency that the GallA-GR59/60AA mutant NTF2 was less efficiently reduced at lower levels of SPPL2a (Figure 9B). This could suggest a minor additional impairment of cleavage efficiency induced by the GallA mutation in the context of the GR59/60AA mutant. However, this effect was very subtle and overcome by increasing amounts of 
SPPL2a. Therefore, the effect of the GR59/60AA exchange seems to stronger than that of the GallA mutation.

Thus, we conclude that the residues 59-60 of murine CD74 are an important primary-structure determinant involved in facilitating the intramembrane cleavage by SPPL2a (Figure 9D). In addition, several glycine residues within the transmembrane segment, most likely based on their helix-destabilizing potential [40], support this process. All these residues identified by our analysis of murine CD74 are conserved in human CD74 (Figure 9E) further underlying their relevance. However, none of these and of all other features analyzed were found to be absolutely essential for cleavage of CD74 by SPPL2a, since processing of the respective mutants occurred with reduced efficiency, but was not entirely abolished. 


\section{DISCUSSION}

Based on our results, we conclude that several determinants within the CD74 luminal membrane-proximal domain and the transmembrane segment act in concert in order to facilitate and promote cleavage by SPPL2a. Since this study describes the first structure-function analysis for substrate cleavage by SPPL2a, a direct comparisons to previous reports is not possible. The most closely related studies have been performed on the homologous protease SPPL2b and its substrate ITM2B (Bri2) [38,41], a protein subjected to ectodomain shedding by ADAM10 prior to the intramembrane cleavage [12]. For ITM2B, the length of the ectodomain was reported to be an important determinant of the cleavage efficiency by SPPL $2 b$ since interference with the release of the ectodomain substantially impaired this process [41]. In general, our results on CD74 support this concept. The CD74 full-length protein was not modulated by coexpressed SPPL2a in MelJuso cells indicating a general requirement of ectodomain trimming. Furthermore, among the different NTFs, we observed an inverse correlation between fragment size and SPPL2a cleavage efficiency. Though primarily the smaller NTF2 was depleted by SPPL2a co-expression, also less pronounced modulation of NTF1 was repeatedly observed. CD74 binds to MHCII via its CLIP segment which may still be preserved in the longer NTF1. Therefore, it may be difficult to distinguish, to what degree the less efficient cleavage of NTF1 is based on the length of its ectodomain or its MHCII association which might also sterically impair the intramembrane cleavage. In SPPL2a-deficient B cells, a distinct CD74 NTF accumulates which is represented in a single band upon Western blot analysis [3]. This suggests a model that under physiological conditions the truncation of the CD74 ectodomain by luminal proteases can only proceed to a certain point and that further degradation of the remaining fragment can only occur by SPPL2a-mediated proteolysis.

For ITM2B, extensive domain-swapping experiments with the non-cleavable homologue ITM2C (Bri3) demonstrated several relevant structural determinants in different parts of the protein in addition to the length of the ectodomain [41]. As mentioned above, generation of chimeric proteins consisting of CD74 and other transmembrane proteins was only successful for an exchange of the ICD by cytoplasmic domains with corresponding endosomal targeting motifs, as depicted in Figure 3. An exchange of the transmembrane segment consistently resulted in ER retention of the chimeric proteins, presumably indicating misfolding and, thereby, precluding any assessment of their cleavability. However, important structural determinants within ITM2B for cleavage by SPPL2b appear to be localized in the transmembrane segment as well as the membrane-proximal regions on both sides of the membrane [41]. At the molecular level, the required features within the juxta-membrane parts of ITM2B have not been precisely explored yet. In the transmembrane segment, the $\alpha$-helical content of this sequence stretch, which is critically influenced by one particular glycine residue, plays an important role [38]. Alanine substitution of this glycine residue significantly impairs cleavage of ITM2B by SPPL2b. As a consensus between our data and the described studies on ITM2B, it can be concluded that multiple sequence determinants contribute to the cleavage of a certain type 2 transmembrane protein by SPPL2 proteases. Similar to ITM2B, we found a role of helix-destabilizing glycine residues within the transmembrane segment of CD74 and of features in the luminal membrane-proximal domain. A difference between both substrates may be that we did not detect a major role of sequence determinants within the CD74 ICD in promoting cleavage by SPPL2a. However, certain features like the palmitoylated cysteine C27 have an impact on properties of the CD74 NTF as discussed below.

Also for other GxGD intramembrane proteases the current understanding of substrate characteristics is limited [2]. The exchange of helix-breaking residues was reported to impair cleavage of signal peptides by SPP [42]. Furthermore, processing of an internal signal sequence of the hepatitis C virus core protein was abolished by specific mutations within this segment indicating that in this case single residues play an essential role [43-46]. Also for cleavage by $\gamma$-secretase a short ectodomain was found to be important [47]. However, this was not generally sufficient to induce cleavage by $\gamma$-secretase arguing for additional substrate determinants, which remain incompletely understood to date [48]. Extensive mutagenesis studies have been performed on different substrates, including the amyloid precursor protein [49-51]. However, only in a few exceptional cases single amino acid exchanges were found to abolish cleavage by $\gamma$-secretase, as it was observed for the Notch1 V744G mutation [52]. In 
contrast to SPP/SPPL proteases, more than sixty $\gamma$-secretase substrates were reported to date [53]. Based on these, no obvious primary-structure encoded consensus sequence could be revealed $[48,53]$. This may indicate that the residues in direct vicinity to the cleavage site are not of particular importance for substrate recognition. Correspondingly, alanine substitution of the residues flanking the main primary cleavage site of CD74 (Y52, F53) did not impair cleavability. This is in contrast to rhomboids, which are serine intramembrane proteases, where a specific rhomboid recognition motif surrounds the cleavage site [54]. In addition, helix-breaking residues within the transmembrane segment of the substrate protein were found to be important also for these proteases [55].

Here we report an unbiased identification of the primary SPPL2a cleavage site within the CD74 NTF. Among other SPPL2 substrates, only the cleavage sites of SPPL2b within TNF $\alpha$ and the transferrin receptor have been reported so far $[2,10,20]$. In all cases, the primary cleavage was found to occur within the outer third of the transmembrane segment facing the organelle lumen/extracellular milieu. An intramembrane cleavage of CD74 that leads to the release of a CD74 ICD was reported prior to the identification of the responsible protease [16]. In that study, leucine 42 was suggested to represent the Cterminus of the liberated ICD. For TNF $\alpha$, the $\mathrm{N}$ - and C-termini of the identified C-peptides and ICDs did not match directly $[2,10]$. Based on this, a sequential cleavage mechanism of SPPL2 proteases which commences with the release of the C-peptide has been proposed [2]. Therefore, our identification of residues 52/53 as site for the release of the CD74 C-peptide could well be compatible with the previously suggested ICD comprising residues up to L42.

Though multiple determinants are involved, our results indicate that particularly the luminal membrane-proximal segment of CD74 plays a role in substrate recognition by the protease SPPL2a. In the $\gamma$-secretase complex, the co-factor nicastrin has been suggested to act as a substrate receptor that controls substrate selection and access to the catalytic subunits. However, according to the current view, SPPL proteases are functional without accessory proteins in contrast to the presenilins, the catalytically active subunits of the $\gamma$-secretase complex [2]. Therefore, a direct interaction between the CD74 membraneproximal segment and the protease SPPL2a may be hypothesized. Within the topology of SPPL2a [56], only the loop between transmembrane segments 6 and 7, which harbor the YD and GxGD motifs, and the glycosylated N-terminal domain with a size of $\sim 150$ amino acids have considerable length that would allow a significant protrusion into the luminal space. Therefore, these parts of the protein may represent candidate regions within the protease to be involved in this interaction.

The physiological function of palmitoylation in integral membrane proteins is currently incompletely understood [57]. In many cases, acylation was suggested to selectively increase the affinity of the proteins to distinct membrane microdomains or lipid rafts. In the endosomal system, palmitoylation has been found to determine the half-life of several membrane proteins [58,59] since loss of the lipid anchor significantly enhanced their turnover. In this regard, the low steady-state NTF levels of nonpalmitoylated CD74 that we observed could be due to enhanced turnover of this fragment, possibly utilizing SPPL2a independent pathways. For selected $\gamma$-secretase substrates, like the p75 neurotrophin receptor, palmitoylation of the substrate was reported to be a prerequisite for the intramembrane cleavage [60]. Our data suggest that palmitoylation of CD74 is per se not required for its proteolysis by SPPL2a. However, due to the low steady-state NTF levels of palmitoylation-deficient CD74, an assessment of the cleavage efficiency appears to be difficult with the risk of not detecting minor reductions. Our observations with the CD74 C27A mutant consistently reflect findings for non-palmitoylated human TNF $\alpha$ [61]. Also for the TNF NTF, the general cleavability of the non-lipidated protein by SPPL2b was preserved. However, enhanced degradation of the NTF by SPPL2b-independent mechanisms resulted in significantly reduced steady-state NTF levels [61].

In summary, we conclude that none of the analyzed primary-structure encoded elements is absolutely indispensable for the intramembrane cleavage of CD74. However, we demonstrated that determinants within the transmembrane segment and especially the luminal membrane-proximal segment are important for this process. How these parts of the substrate are precisely involved in facilitating recognition and processing by SPPL2a will require further studies. In general, a deeper understanding of the substrate requirements of SPPL2a and other SPPL proteases will greatly benefit from similar mutagenesis approaches with additional substrates in order to recognize similarities between the different 
proteins. In light of the limited number of currently known substrates of SPPL2a, the identification of additional substrates will be a prerequisite to provide a broader basis for these studies.

\section{AUTHOR CONTRIBUTION}

S.H., F.H. T.M., S.H., A.F. performed the experiments and analysed the data. B.S. and R.F. designed, conceptualised and supervised the research. H.S. and P.S. gave conceptual advice. B.S. wrote the manuscript. All authors contributed to the editing of the manuscript.

\section{CONFLICT OF INTEREST}

The authors declare that they have no conflicts of interest.

\section{ACKNOWLEDGEMENTS}

We thank Jacques Neefjes, Netherlands Cancer Institute, Amsterdam, for the generous gift of MelJuso cells and Martina Haug-Kröper for excellent technical assistance.

\section{FUNDING}

This work was supported by the Deutsche Forschungsgemeinschaft as part of the SFB877 (project B7, to B.S.), the Cluster of Excellence "Inflammation at Interfaces" as well as grants SCHR 1284/1-1 (to B.S.) and FL 635/2-1 (to R.F.). 


\section{REFERENCES}

1 Jurisch-Yaksi, N., Sannerud, R., and Annaert, W. (2013) A fast growing spectrum of biological functions of gamma-secretase in development and disease. Biochim. Biophys. Acta 1828, 28152827

2 Voss, M., Schröder, B., and Fluhrer, R. (2013) Mechanism, specificity, and physiology of signal peptide peptidase (SPP) and SPP-like proteases. Biochim. Biophys. Acta 1828, 2828-2839

3 Schneppenheim, J., Dressel, R., Hüttl, S., Lüllmann-Rauch, R., Engelke, M., Dittmann, K., Wienands, J., Eskelinen, E. L., Hermans-Borgmeyer, I., Fluhrer, R., Saftig, P., and Schröder, B. (2013) The intramembrane protease SPPL2a promotes B cell development and controls endosomal traffic by cleavage of the invariant chain. J. Exp. Med. 210, 41-58

4 Beisner, D. R., Langerak, P., Parker, A. E., Dahlberg, C., Otero, F. J., Sutton, S. E., Poirot, L., Barnes, W., Young, M. A., Niessen, S., Wiltshire, T., Bodendorf, U., Martoglio, B., Cravatt, B., and Cooke, M. P. (2013) The intramembrane protease Sppl2a is required for B cell and DC development and survival via cleavage of the invariant chain. J. Exp. Med. 210, 23-30

5 Bergmann, H., Yabas, M., Short, A., Miosge, L., Barthel, N., Teh, C. E., Roots, C. M., Bull, K. R., Jeelall, Y., Horikawa, K., Whittle, B., Balakishnan, B., Sjollema, G., Bertram, E. M., MacKay, F., Rimmer, A. J., Cornall, R. J., Field, M. A., Andrews, T. D., Goodnow, C. C., and Enders, A. (2013) B cell survival, surface BCR and BAFFR expression, CD74 metabolism, and CD8dendritic cells require the intramembrane endopeptidase SPPL2A. J. Exp. Med. 210, 31-40

6 Hüttl, S., Kläsener, K., Schweizer, M., Schneppenheim, J., Oberg, H. H., Kabelitz, D., Reth, M., Saftig, P., and Schröder, B. (2015) Processing of CD74 by the Intramembrane Protease SPPL2a Is Critical for B Cell Receptor Signaling in Transitional B Cells. J. Immunol. 195, 1548-1563

7 Behnke, J., Schneppenheim, J., Koch-Nolte, F., Haag, F., Saftig, P., and Schröder, B. (2011) Signal-peptide-peptidase-like 2a (SPPL2a) is targeted to lysosomes/late endosomes by a tyrosine motif in its C-terminal tail. FEBS Lett. 585, 2951-2957

8 Friedmann, E., Hauben, E., Maylandt, K., Schleeger, S., Vreugde, S., Lichtenthaler, S. F., Kuhn, P. H., Stauffer, D., Rovelli, G., and Martoglio, B. (2006) SPPL2a and SPPL2b promote intramembrane proteolysis of TNFalpha in activated dendritic cells to trigger IL-12 production. Nat. Cell Biol. 8, 843-848

9 Schneppenheim, J., Hüttl, S., Mentrup, T., Lüllmann-Rauch, R., Rothaug, M., Engelke, M., Dittmann, K., Dressel, R., Araki, M., Araki, K., Wienands, J., Fluhrer, R., Saftig, P., and Schröder, B. (2014) The intramembrane proteases signal Peptide peptidase-like $2 \mathrm{a}$ and $2 \mathrm{~b}$ have distinct functions in vivo. Mol. Cell Biol. 34, 1398-1411

10 Fluhrer, R., Grammer, G., Israel, L., Condron, M. M., Haffner, C., Friedmann, E., Bohland, C., Imhof, A., Martoglio, B., Teplow, D. B., and Haass, C. (2006) A gamma-secretase-like intramembrane cleavage of TNFalpha by the GxGD aspartyl protease SPPL2b. Nat. Cell Biol. 8, 894-896

11 Kirkin, V., Cahuzac, N., Guardiola-Serrano, F., Huault, S., Luckerath, K., Friedmann, E., Novac, N., Wels, W. S., Martoglio, B., Hueber, A. O., and Zornig, M. (2007) The Fas ligand intracellular domain is released by ADAM10 and SPPL2a cleavage in T-cells. Cell Death. Differ. 14, 16781687

12 Martin, L., Fluhrer, R., Reiss, K., Kremmer, E., Saftig, P., and Haass, C. (2008) Regulated intramembrane proteolysis of Bri2 (Itm2b) by ADAM10 and SPPL2a/SPPL2b. J. Biol. Chem. 283, 1644-1652

13 Brady, O. A., Zhou, X., and Hu, F. (2014) Regulated Intramembrane Proteolysis of the Frontotemporal Lobar Degeneration Risk Factor, TMEM106B, by Signal Peptide Peptidase-like 2a (SPPL2a). J. Biol. Chem. 289, 19670-19680

14 Bird, P. I., Trapani, J. A., and Villadangos, J. A. (2009) Endolysosomal proteases and their inhibitors in immunity. Nat. Rev. Immunol. 9, 871-882 
15 Colbert, J. D., Matthews, S. P., Miller, G., and Watts, C. (2009) Diverse regulatory roles for lysosomal proteases in the immune response. Eur. J. Immunol. 39, 2955-2965

16 Matza, D., Kerem, A., Medvedovsky, H., Lantner, F., and Shachar, I. (2002) Invariant chaininduced B cell differentiation requires intramembrane proteolytic release of the cytosolic domain. Immunity. 17, 549-560

17 Mentrup, T., Hasler, R., Fluhrer, R., Saftig, P., and Schröder, B. (2015) A Cell-Based Assay Reveals Nuclear Translocation of Intracellular Domains Released by SPPL Proteases. Traffic. 16, 871-892

18 Schröder, B., Wrocklage, C., Hasilik, A., and Saftig, P. (2010) Molecular characterisation of 'transmembrane protein 192' (TMEM192), a novel protein of the lysosomal membrane. Biol Chem. 391, 695-704

19 Savalas, L. R., Gasnier, B., Damme, M., Lubke, T., Wrocklage, C., Debacker, C., Jezegou, A., Reinheckel, T., Hasilik, A., Saftig, P., and Schröder, B. (2011) Disrupted in renal carcinoma 2 (DIRC2), a novel transporter of the lysosomal membrane, is proteolytically processed by cathepsin L. Biochem. J. 439, 113-128

20 Zahn, C., Kaup, M., Fluhrer, R., and Fuchs, H. (2013) The transferrin receptor-1 membrane stub undergoes intramembrane proteolysis by signal peptide peptidase-like 2b. FEBS J. 280, 16531663

21 Schagger, H. and Von, J. G. (1987) Tricine-sodium dodecyl sulfate-polyacrylamide gel electrophoresis for the separation of proteins in the range from 1 to $100 \mathrm{kDa}$. Anal. Biochem. 166, 368-379

22 Laemmli, U. K. (1970) Cleavage of structural proteins during the assembly of the head of bacteriophage T4. Nature 227, 680-685

23 Koch, N., Koch, S., and Hammerling, G. J. (1982) Ia invariant chain detected on lymphocyte surfaces by monoclonal antibody. Nature 299, 644-645

24 Ashman, J. B. and Miller, J. (1999) A role for the transmembrane domain in the trimerization of the MHC class II-associated invariant chain. J. Immunol. 163, 2704-2712

25 Wubbolts, R., Fernandez-Borja, M., Oomen, L., Verwoerd, D., Janssen, H., Calafat, J., Tulp, A., Dusseljee, S., and Neefjes, J. (1996) Direct vesicular transport of MHC class II molecules from lysosomal structures to the cell surface. J. Cell Biol. 135, 611-622

26 Landsverk, O. J., Bakke, O., and Gregers, T. F. (2009) MHC II and the endocytic pathway: regulation by invariant chain. Scand. J. Immunol. 70, 184-193

27 Lagaudriere-Gesbert, C., Newmyer, S. L., Gregers, T. F., Bakke, O., and Ploegh, H. L. (2002) Uncoating ATPase Hsc70 is recruited by invariant chain and controls the size of endocytic compartments. Proc. Natl. Acad. Sci. U. S. A 99, 1515-1520

28 Nordeng, T. W., Gregers, T. F., Kongsvik, T. L., Meresse, S., Gorvel, J. P., Jourdan, F., Motta, A., and Bakke, O. (2002) The cytoplasmic tail of invariant chain regulates endosome fusion and morphology. Mol. Biol. Cell 13, 1846-1856

29 Stang, E. and Bakke, O. (1997) MHC class II-associated invariant chain-induced enlarged endosomal structures: a morphological study. Exp. Cell Res. 235, 79-92

30 Matza, D., Kerem, A., and Shachar, I. (2003) Invariant chain, a chain of command. Trends Immunol. 24, 264-268

31 Becker-Herman, S., Arie, G., Medvedovsky, H., Kerem, A., and Shachar, I. (2005) CD74 is a member of the regulated intramembrane proteolysis-processed protein family. Mol. Biol. Cell 16, 5061-5069

32 Voss, M., Kunzel, U., Higel, F., Kuhn, P. H., Colombo, A., Fukumori, A., Haug-Kroper, M., Klier, B., Grammer, G., Seidl, A., Schroder, B., Obst, R., Steiner, H., Lichtenthaler, S. F., Haass, C., and Fluhrer, R. (2014) Shedding of glycan-modifying enzymes by signal peptide peptidaselike 3 (SPPL3) regulates cellular N-glycosylation. EMBO J. 33, 2890-2905

33 Bonifacino, J. S. and Traub, L. M. (2003) Signals for sorting of transmembrane proteins to endosomes and lysosomes. Annu. Rev. Biochem. 72, 395-447 
34 Behnke, J., Eskelinen, E. L., Saftig, P., and Schröder, B. (2011) Two dileucine motifs mediate late endosomal/lysosomal targeting of transmembrane protein 192 (TMEM192) and a C-terminal cysteine residue is responsible for disulfide bond formation in TMEM192 homodimers. Biochem. J. 434, 219-231

35 Simonis, S. and Cullen, S. E. (1986) Fatty acylation of murine Ia alpha, beta, and invariant chains. J. Immunol. 136, 2962-2967

36 Koch, N. and Hammerling, G. J. (1986) The HLA-D-associated invariant chain binds palmitic acid at the cysteine adjacent to the membrane segment. J. Biol. Chem. 261, 3434-3440

37 Koch, N. (1988) Posttranslational modifications of the Ia-associated invariant protein p41 after gene transfer. Biochemistry 27, 4097-4102

38 Fluhrer, R., Martin, L., Klier, B., Haug-Kroper, M., Grammer, G., Nuscher, B., and Haass, C. (2012) The alpha-helical content of the transmembrane domain of the British dementia protein-2 (Bri2) determines its processing by signal peptide peptidase-like 2b (SPPL2b). J. Biol. Chem. 287, 5156-5163

39 Driessen, C., Bryant, R. A., Lennon-Dumenil, A. M., Villadangos, J. A., Bryant, P. W., Shi, G. P., Chapman, H. A., and Ploegh, H. L. (1999) Cathepsin S controls the trafficking and maturation of MHC class II molecules in dendritic cells. J. Cell Biol. 147, 775-790

40 Pace, C. N. and Scholtz, J. M. (1998) A helix propensity scale based on experimental studies of peptides and proteins. Biophys. J. 75, 422-427

41 Martin, L., Fluhrer, R., and Haass, C. (2009) Substrate requirements for SPPL2b-dependent regulated intramembrane proteolysis. J. Biol. Chem. 284, 5662-5670

42 Lemberg, M. K. and Martoglio, B. (2002) Requirements for signal peptide peptidase-catalyzed intramembrane proteolysis. Mol. Cell 10, 735-744

43 Oehler, V., Filipe, A., Montserret, R., da, C. D., Brown, G., Penin, F., and McLauchlan, J. (2012) Structural analysis of hepatitis $\mathrm{C}$ virus core-E1 signal peptide and requirements for cleavage of the genotype 3a signal sequence by signal peptide peptidase. J. Virol. 86, 7818-7828

44 Okamoto, K., Mori, Y., Komoda, Y., Okamoto, T., Okochi, M., Takeda, M., Suzuki, T., Moriishi, K., and Matsuura, Y. (2008) Intramembrane processing by signal peptide peptidase regulates the membrane localization of hepatitis $\mathrm{C}$ virus core protein and viral propagation. J. Virol. 82, 83498361

45 McLauchlan, J., Lemberg, M. K., Hope, G., and Martoglio, B. (2002) Intramembrane proteolysis promotes trafficking of hepatitis C virus core protein to lipid droplets. EMBO J. 21, 3980-3988

46 Ma, H. C., Ku, Y. Y., Hsieh, Y. C., and Lo, S. Y. (2007) Characterization of the cleavage of signal peptide at the $\mathrm{C}$-terminus of hepatitis $\mathrm{C}$ virus core protein by signal peptide peptidase. $\mathrm{J}$. Biomed. Sci. 14, 31-41

47 Struhl, G. and Adachi, A. (2000) Requirements for presenilin-dependent cleavage of notch and other transmembrane proteins. Mol. Cell 6, 625-636

48 Hemming, M. L., Elias, J. E., Gygi, S. P., and Selkoe, D. J. (2008) Proteomic profiling of gammasecretase substrates and mapping of substrate requirements. PLoS. Biol. 6, e257

49 Lichtenthaler, S. F., Wang, R., Grimm, H., Uljon, S. N., Masters, C. L., and Beyreuther, K. (1999) Mechanism of the cleavage specificity of Alzheimer's disease gamma-secretase identified by phenylalanine-scanning mutagenesis of the transmembrane domain of the amyloid precursor protein. Proc. Natl. Acad. Sci. U. S. A 96, 3053-3058

50 Lichtenthaler, S. F., Ida, N., Multhaup, G., Masters, C. L., and Beyreuther, K. (1997) Mutations in the transmembrane domain of APP altering gamma-secretase specificity. Biochemistry 36, 1539615403

51 Ren, Z., Schenk, D., Basi, G. S., and Shapiro, I. P. (2007) Amyloid beta-protein precursor juxtamembrane domain regulates specificity of gamma-secretase-dependent cleavages. J. Biol. Chem. 282, 35350-35360

52 Huppert, S. S., Le, A., Schroeter, E. H., Mumm, J. S., Saxena, M. T., Milner, L. A., and Kopan, R. (2000) Embryonic lethality in mice homozygous for a processing-deficient allele of Notch1. Nature 405, 966-970 
53 Beel, A. J. and Sanders, C. R. (2008) Substrate specificity of gamma-secretase and other intramembrane proteases. Cell Mol. Life Sci. 65, 1311-1334

54 Strisovsky, K., Sharpe, H. J., and Freeman, M. (2009) Sequence-specific intramembrane proteolysis: identification of a recognition motif in rhomboid substrates. Mol. Cell 36, 1048-1059

55 Urban, S. and Freeman, M. (2003) Substrate specificity of rhomboid intramembrane proteases is governed by helix-breaking residues in the substrate transmembrane domain. Mol. Cell 11, 14251434

56 Friedmann, E., Lemberg, M. K., Weihofen, A., Dev, K. K., Dengler, U., Rovelli, G., and Martoglio, B. (2004) Consensus analysis of signal peptide peptidase and homologous human aspartic proteases reveals opposite topology of catalytic domains compared with presenilins. J. Biol. Chem. 279, 50790-50798

57 Blaskovic, S., Blanc, M., and van der Goot, F. G. (2013) What does S-palmitoylation do to membrane proteins? FEBS J. 280, 2766-2774

58 Rohrer, J., Schweizer, A., Johnson, K. F., and Kornfeld, S. (1995) A determinant in the cytoplasmic tail of the cation-dependent mannose 6-phosphate receptor prevents trafficking to lysosomes. J. Cell Biol. 130, 1297-1306

59 McCormick, P. J., Dumaresq-Doiron, K., Pluviose, A. S., Pichette, V., Tosato, G., and Lefrancois, S. (2008) Palmitoylation controls recycling in lysosomal sorting and trafficking. Traffic. 9, 19841997

60 Underwood, C. K., Reid, K., May, L. M., Bartlett, P. F., and Coulson, E. J. (2008) Palmitoylation of the C-terminal fragment of p75(NTR) regulates death signaling and is required for subsequent cleavage by gamma-secretase. Mol. Cell Neurosci. 37, 346-358

61 Poggi, M., Kara, I., Brunel, J. M., Landrier, J. F., Govers, R., Bonardo, B., Fluhrer, R., Haass, C., Alessi, M. C., and Peiretti, F. (2013) Palmitoylation of TNF alpha is involved in the regulation of TNF receptor 1 signalling. Biochim. Biophys. Acta 1833, 602-612 


\section{FIGURE LEGENDS}

Figure $1 \quad$ CD74 is sequentially processed in the melanoma cell line MelJuso.

(A) Model depicting the current knowledge of CD74 proteolysis after delivering MHCII complexes (MHCII) to endosomal compartments. There, different endosomal proteases including cathepsin S (CTSS) degrade the CD74 luminal domain generating an N-terminal fragment (NTF) which is further processed by SPPL2a. Finally, an intracellular domain (ICD) and a C-terminal cleavage fragment (C-peptide) are released. The position of the epitope of the employed monoclonal In-1 antibody has been marked. (B) Upon co-expression of murine CD74 and SPPL2a in MelJuso cells, substrate and protease co-localized as visualized by indirect immunofluorescence using the In-1 antibody directed against an N-terminal epitope of CD74. Detection of SPPL2a was achieved using an antibody against the C-terminally fused Myc epitope. Scale bars, $5 \mu \mathrm{m}$. (C) MelJuso cells were transfected with siRNA against SPPL2a or a nontargeting control siRNA. Total cell lysates were analyzed by Western blotting for endogenous SPPL2a and CD74. The PIN1 antibody utilized for detection of human CD74 was directed against an N-terminal epitope. (D, E) Delivery to post-ER compartments is required for processing of CD74. MelJuso cells transiently expressing CD74-HA were treated for $18 \mathrm{~h}$ with brefeldin A $(1 \mu \mathrm{g} / \mathrm{ml})$ or solvent prior to analysis by Western blot (D) or indirect immunofluorescence (E). Upon Western blot analysis with the In1 antibody, the CD74 full-length protein (FL) and different N-terminal fragments (NTF1, NTF2), which were absent in brefeldin A treated cells, were detected. ER retention of CD74 following brefeldin A application was confirmed by its co-localization with the ER marker protein, protein disulfide isomerase (PDI). Scale bars, $5 \mu \mathrm{m}$. (F) mCD74-HA was transiently expressed either alone or in combination with catalytically active or inactive SPPL2a (D416A) in MelJuso cells. Co-expressed SPPL2a particularly induced depletion of the CD74 NTF2. The antibodies employed for the detection of murine CD74 (mCD74) and murine SPPL2a (mSPPL2a) did not cross-react with the corresponding human proteins being present endogenously in MelJuso cells. (G) The capability of endogenous SPPL2a to process the heterologously expressed murine CD74 (mCD74-HA) was analyzed in MelJuso cells transfected with siRNA against SPPL2a or a non-targeting control. *, unspecific band.

\section{Figure 2 Determination of the SPPL2a cleavage site within CD74}

(A) Schematic presentation of the epitope-tagged CD74 N-terminal fragment (NTF) model substrate comprising amino acids 2 to 82 of the CD74 p31 protein and an N-terminal V5 as well as a C-terminal Flag epitope tag. Amino acids are depicted using the one letter code. The predicted transmembrane domain is illustrated in grey. At the very $\mathrm{C}$-terminus an alanine-proline motif was added to prevent degradation of liberated fragments by exopeptidases. (B) Mass spectrometric analysis of CD74 NTF cleavage products secreted into conditioned supernatants of HEK293 cells (first panel) or HEK293 cells stably transfected with SPPL2a (second \& third panel). CD74 was transiently expressed in the indicated cell lines. Where indicated, cells were treated with (Z-LL) 2 -ketone for $18 \mathrm{~h}$ prior to cell harvest. Mass spectrometric analysis revealed six peptides (numbered arrowheads) that mapped to the CD74 NTF. The very same peptides were more abundant in conditioned supernatants of SPPL2a-overexpressing cells but reduced upon concomitant treatment with (Z-LL) 2 -ketone. (C) Overview of peptide fragments assigned to the peaks detected. obs. mass, observed mass; calc. mass, calculated mass.

\section{Figure 3 Exchange of the CD74 cytoplasmic domain does not impair cleavage by SPPL2a.}

(A) Scheme of the employed DIRC2 $2_{\mathrm{NT}}-\mathrm{mCD} 74$ and TMEM192 $2_{\mathrm{NT}}-\mathrm{mCD} 74$ chimeric proteins. The cytoplasmic $\mathrm{N}$-terminal segment of the lysosomal multipass membrane proteins DIRC2 (aa 2-52; yellow) 
and TMEM192 (aa 2-47; orange) which contain established dileucine-based lysosomal sorting signals (LL or LI, respectively) were fused to the transmembrane segment (TM; brown) and luminal domain of the murine CD74 p31 (CD74; green) isoform. The CLIP (class II-associated li chain peptide; purple) segment which represents the main interaction site between CD74 and MHCII was preserved in all three constructs. Furthermore, all constructs exhibited N-terminal HA and C-terminal V5 tags in order to facilitate detection. (B) Lysosomal/endosomal targeting of the DIRC2 $2_{\mathrm{NT}}-\mathrm{mCD} 74$ and TMEM192 $2_{\mathrm{NT}}$ mCD74 proteins. Both constructs were transiently expressed in HeLa cells together with SPPL2a carrying a Myc epitope at its C-terminus. Co-localization of the chimeric proteins with the co-expressed protease was analyzed in comparison to wild type CD74 (HA-mCD74-V5) by indirect immunofluorescence via detection of the HA (CD74, chimeras) and Myc (SPPL2a) epitopes. Scale bars, $5 \mu \mathrm{m}$. (C) Proteolytic processing of the DIRC2 $2_{\mathrm{NT}}-\mathrm{mCD} 74$ and TMEM192 $2_{\mathrm{NT}}-\mathrm{mCD} 74$ chimeras upon transient expression in HeLa cells. SPPL2a or the catalytically inactive SPPL2a-D416A (D/A) mutant were co-expressed with the indicated substrates. Total cell lysates were analyzed by Western blotting for full-length CD74 (FL) and the different NTFs (NTF1, NTF2) of CD74 and the respective CD74 chimeric proteins.

\section{Figure 4 Systematic alanine-scanning mutagenesis of the CD74 NTF}

(A) Overview of the generated alanine replacement mutants within the cytoplasmic, transmembrane and luminal membrane-proximal domains of CD74. All expression constructs were based on the murine p31 isoform and exhibited an HA epitope fused to the 3' end of the CD74 open reading frame. CD74 has been reported to undergo palmitoylation at the membrane-proximal cysteine residue $\mathrm{C} 27$ as depicted. (B) Processing of the CD74 mutants was analyzed upon transient expression in MelJuSo cells. CD74 wild type or mutant expression constructs were transfected alone or in combination with active or catalytically inactive SPPL2a (D/A) into MelJuso cells. Western blot of total cell lysates was performed for CD74 with an antibody against an N-terminal epitope detecting the CD74 full-length protein (FL) and the different $\mathrm{N}$ terminal fragments (NTF1, NTF2). As indicated, expression of the proteases and equal protein loading were controlled using antibodies against SPPL2a, EEF and GAPDH, respectively. In the upper row, expression of the 18-23A construct was confirmed by detection of the C-terminal HA epitope. (C) Reanalysis of the 18-23A exchange mutant using an expression construct with an $\mathrm{N}$-terminal $\mathrm{HA}$ and a Cterminal V5 epitope. Processing of this mutant was examined in transiently transfected MelJuSo cells as described above in comparison to equivalently tagged wild type CD74 (HA-mCD74-V5).

Figure 5 Palmitoylation-deficient CD74 exhibits lower steady-state NTF levels, but can be cleaved by SPPL2a.

(A) Indirect immunofluorescence analysis of MelJuso cells transiently expressing the non-palmitoylatable CD74 C27A mutant and SPPL2a. Visualization of CD74 and SPPL2a was achieved using the In-1 antibody against CD74 and anti-Myc. Scale bars, $5 \mu \mathrm{m}$. (B) Proteolytic processing of the CD74 C27A mutant was compared to that of wild type CD74. Western blot analysis was performed of total lysates of MelJuso cells transiently transfected with the respective CD74 variant alone or together with active or inactive (D/A) SPPL2a.

\section{Figure 6 Single substitutions of residues 38-40, 42-44 and 52-54 do not impair intramembrane cleavage of CD74.}

Based on the poor expression of the 38-40A, 42-44A and 52-54A mutants with an exchange of three amino acids, alanine replacement mutants of single residues from the 38-40 (V38A, L39A, V40A), 42-44 (L42A, L43A, L44A) and 52-54 (Y52A, F53A, L54A) stretches were generated. These CD74 mutant 
proteins were transiently expressed in MelJuSo cells with a C-terminally fused HA epitope. (A) For all mutants, subcellular distribution and co-localization with co-expressed SPPL2a was analyzed by indirect immunofluorescence. Scale bars, $5 \mu \mathrm{m}$. (B-D) Processing of the mutant CD74 NTFs (38-40, B; 42-44, C; $52-54$, D) by co-expressed catalytically active or inactive (D/A) SPPL2a was monitored by Western blot analysis. For detection of the CD74 mutants, an antibody against an N-terminal epitope was employed revealing full-length protein (FL) and NTFs (NTF1, NTF2). EEF2 or actin were detected to confirm equal protein loading.

\section{Figure 7 Helix-destabilizing glycines facilitate CD74 cleavage by SPPL2a but are not essential for this process.}

(A) The transmembrane segment of CD74 exhibits three glycine residues at positions 30, 35 and 46 that were exchanged to alanines resulting in the CD74 GallA mutant. (B) Wild type CD74 (mCD74-HA) and the GallA CD74 mutant were visualized using the In-1 antibody detecting an N-terminal epitope of CD74. Co-expressed SPPL2a was detected with an antibody against the C-terminally fused Myc epitope in order to determine co-localization between CD74 and SPPL2a. Scale bars, $5 \mu \mathrm{m}$. (C) Proteolytic processing of the CD74 GallA mutant was analyzed in comparison to wild type CD74 upon transient expression in MelJuSo cells. Both constructs carried a C-terminal HA epitope. Catalytically active or inactive (D/A) SPPL2a were co-expressed as indicated. Full-length CD74 (FL) and NTFs (NTF1, NTF2) were detected with the In-1 antibody. (D) NTF2 levels from wild type and GallA CD74 were quantified densitometrically and expressed as ratio (\%) between cells co-expressing catalytically active SPPL2a and cells just expressing the substrate CD74. To account for possible differences in transfection efficiency, normalization to the level of the full-length protein in the individual samples (NTF2/FL) was performed before the ratios were calculated. Mean $\pm \mathrm{SD}, \mathrm{n}=3$. (E) Processing of wild type and GallA CD74 by coexpressed SPPL2a was monitored upon additional co-expression of cathepsin S (CTSS) in order to enhance processing of the CD74 luminal domain. To control for differences in protein loading, actin or EEF2 were detected as indicated.

Figure 8 Determinants within the luminal membrane-proximal domain of CD74 facilitate cleavage by SPPL2a.

(A) Wild type CD74 and the 55-60A mutant CD74 were expressed in MelJuSo cells alone or in combination with cathepsin S (CTSS) and/or SPPL2a. Proteolytic processing of CD74 was analyzed with the In-1 antibody revealing the CD74 full-length protein (FL) as well as NTFs (NTF1, NTF2). Processing of NTFs derived from the 55-60A CD74 mutant upon co-expression of SPPL2a appeared to be less complete than for the wild type protein. (B) The SPPL2a-induced reduction of the 55-60A NTF2 is significantly less efficient than of the wild type NTF2. Densitometric quantification of wild type and 5560A NTF2 levels was performed and ratios (\%) between protease-expressing cells (CD74 + SPPL2a) and those just expressing the substrate (CD74) were calculated. Prior to this, the determined values were normalized to the level of the full-length protein in the individual samples (NTF2/FL). Mean $\pm \mathrm{SD}, \mathrm{n}=3$. *, P $<0.05$; unpaired, two-tailed Student's $t$ test. (C-F) In order to further narrow down the relevant determinants within residues 55-60, YQ55/56AA, QQ57/58AA, GR59/60AA mutant CD74 expression constructs were generated exhibiting alanine substitutions of two adjacent residues. (C) Indirect immunofluorescence analysis of MelJuso cells transiently expressing wild type CD74 or the 55-60A, YQ55/56AA, QQ57/58AA or GR59/60AA mutant as well as SPPL2a. CD74 and SPPL2a were visualized with the In-1 antibody and anti-Myc, respectively. Scale bars, $5 \mu \mathrm{m}$. (D) Processing of the YQ55/56AA, QQ57/58AA, GR59/60AA CD74 variants was examined in transiently transfected MelJuso cells upon coexpression of catalytically active or inactive (D/A) SPPL2a. (E) The GR59/60AA mutant NTF2 is significantly less efficiently processed by SPPL2a than the wild type NTF2. The residual NTF2 levels in 
cells overexpressing SPPL2a were determined for the YQ55/56AA, QQ57/58AA, GR59/60AA CD74 mutants in comparison to wild type CD74. Data analysis and quantification of the ratio (\%) were performed as in $(\mathbf{B})$. Mean $\pm \mathrm{SD}, \mathrm{n}=3$. $^{*}, \mathrm{P}<0.05$. Statistical significance was evaluated for the different mutants versus wild type CD74 using an unpaired, two-tailed Student's $t$ test. (F) Co-expression of CTSS was employed in order to enhance processing of the CD74 luminal domain of the YQ55/56AA, QQ57/58AA, GR59/60AACD74 mutants as well as the wild type protein. In (A, D, F) EEF2 was detected in order to control for differences in protein loading.

\section{Figure 9 Several determinants contribute to the cleavability of CD74}

(A) The effect of combining the GallA and GR59/60AA mutations (GallA-GR59/60AA) on CD74 proteolysis was analyzed. Therefore, the GallA-GR59/60AA mutant as well as the GR59/60AA single mutant and wild type CD74 were transiently expressed in MelJuso cells. In order to semi-quantitatively compare cleavability, increasing amounts of SPPL2a expression plasmid were co-transfected. Levels of full-length CD74 (FL) and NTFs (NTF1, NTF2) were analyzed by Western blotting using the In-1 antibody. (B) Densitometric quantification of the representative experiment depicted in (A). NTF2 levels were normalized to the level of the full-length protein in each sample. Then, ratios (\%) between proteaseoverexpressing in comparison to the cells just expressing the substrate were calculated and are depicted. (C) Indirect immunofluorescence analysis of MelJuso cells transiently transfected with wild type CD74 or the GallA-GR59/60AA combination mutant was performed. Murine CD74 and SPPL2a were detected with the In-1 antibody and anti-Myc, respectively. Scale bars, $5 \mu \mathrm{m}$. (D) Scheme of the determinants within the CD74 NTF that were identified to facilitate intramembrane proteolysis by SPPL2a. (E) Alignment of the transmembrane (TM) and membrane-proximal segments of human and murine CD74. The position of the sequence elements identified within this study to enhance cleavability of murine CD74 (blue shading) are conserved within the human protein. 


\section{FIGURE 1}

A
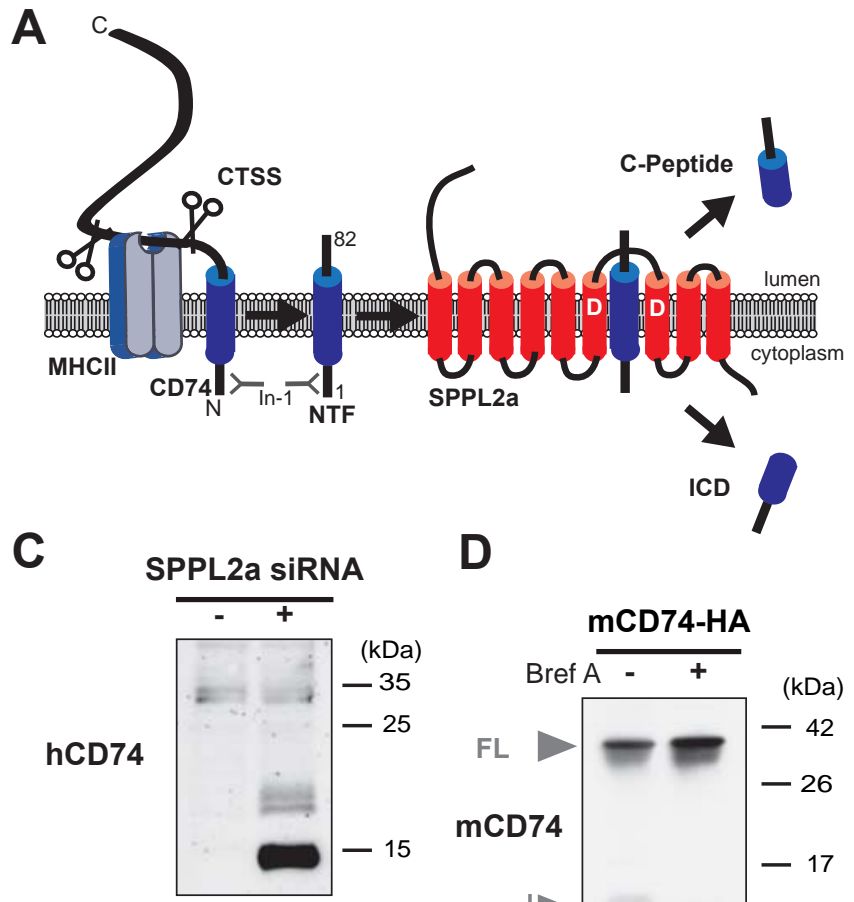

D

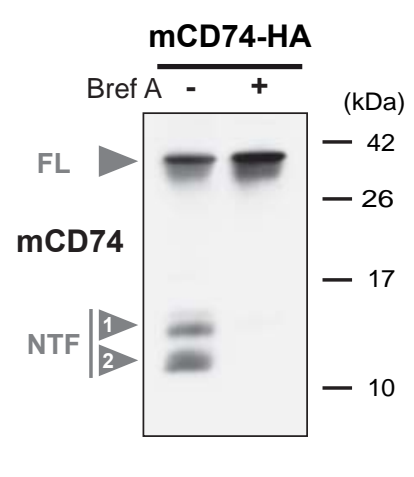

B

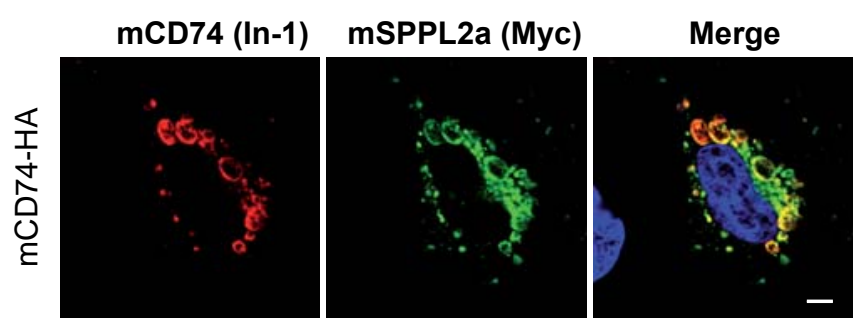

E

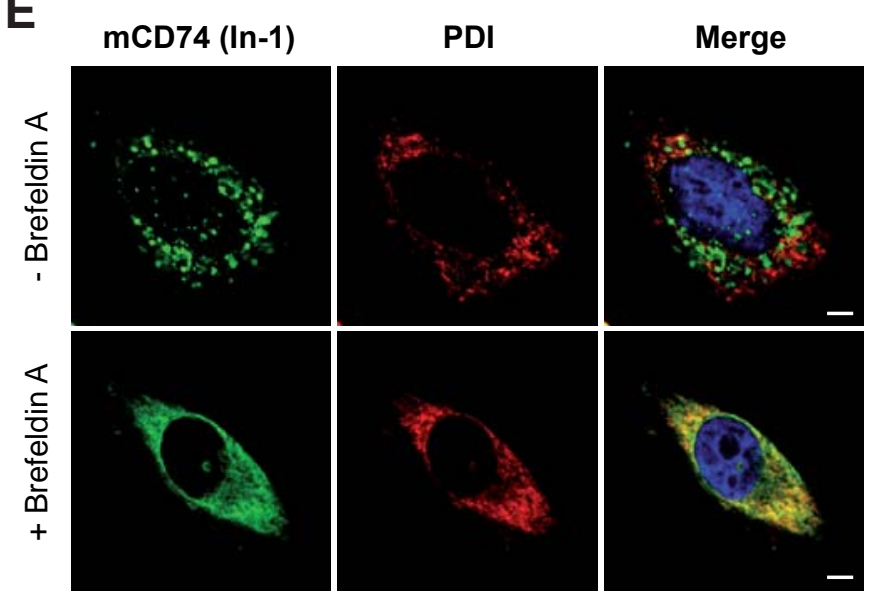

G

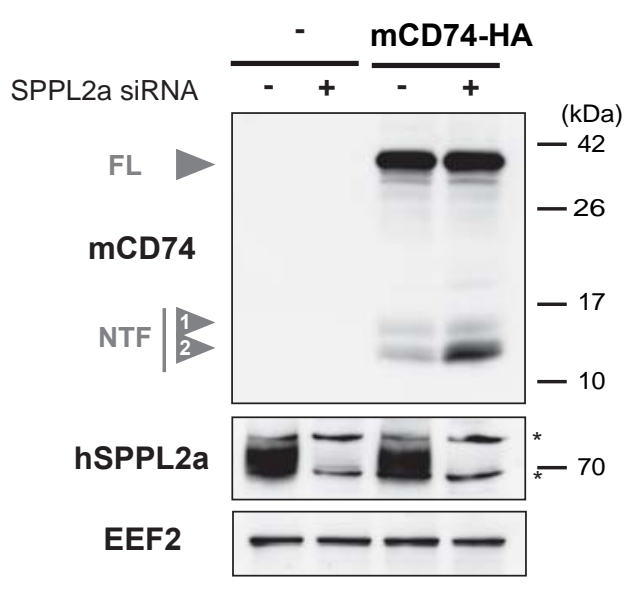




\section{FIGURE 2}

A mCD74 NTF

MGKPIPNPLLGLDSTDDQRDLISNHEQLPILGNRPREPERCSRGALYTGVSVLVALLLAGQATTAYFLYQ

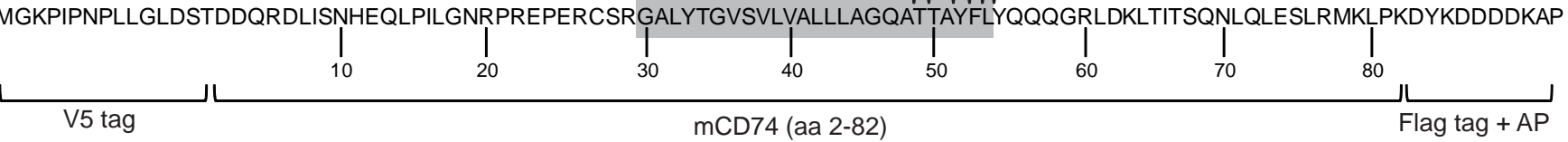

B mCD74 NTF

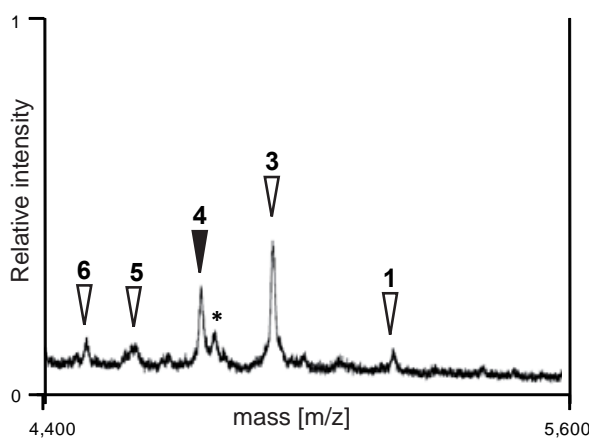

mCD74 NTF + mSPPL2a

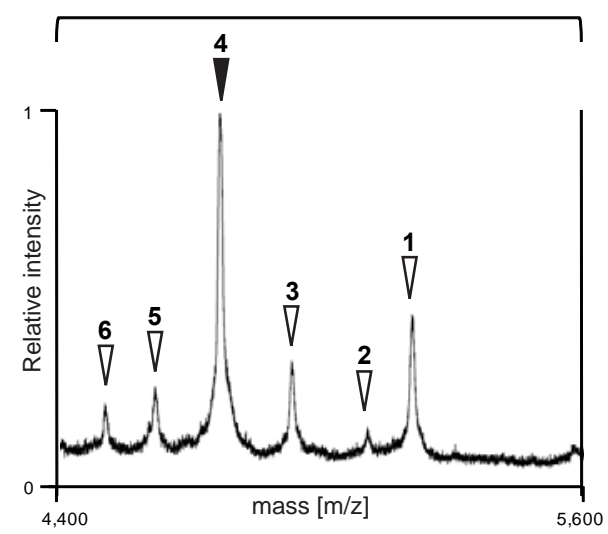
mCD74 NTF + mSPPL2a + (Z-LL)

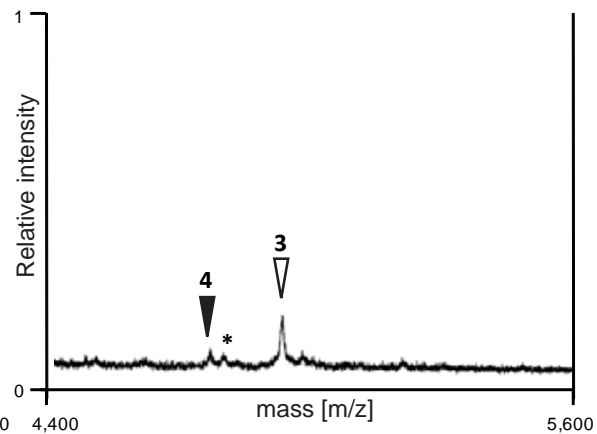

C

\begin{tabular}{|c|c|l|c|c|}
\hline Peak No. & Fragment & Sequence & obs. mass [Da] & calc. mass [Da] \\
\hline 1 & T49 & TTAYFLYQQQGRLDKLTITSQNLQLESLRMKLPKDYKDDDDKAP & 5194 & 5191 \\
\hline 2 & T50 & TAYFLYQQQGRLDKLTITSQNLQLESLRMKLPKDYKDDDDKAP & 5092 & 5090 \\
\hline 3 & Y52 & YFLYQQQGRLDKLTITSQNLQLESLRMKLPKDYKDDDDKAP & 4919 & 4918 \\
\hline 4 & F53 & FLYQQQGRLDKLTITSQNLQLESLRMKLPKDYKDDDDKAP & 4755 & 4754 \\
\hline 5 & L54 & LYQQQGRLDKLTITSQNLQLESLRMKLPKDYKDDDDKAP & 4609 & 4607 \\
\hline 6 & Y55 & YQQQGRLDKLTITSQNLQLESLRMKLPKDYKDDDDKAP & 4494 & 4494 \\
\hline
\end{tabular}




\section{FIGURE 3}

A

\begin{tabular}{|c|c|c|c|c|c|}
\hline HA-mCD74-V5 & & \begin{tabular}{|l|l} 
HA & LI IL \\
\end{tabular} & TM & CLiP & V5 \\
\hline & & $\left|\begin{array}{ll}2 & 29\end{array}\right|$ & & & \\
\hline${ }^{H A-D I R C 2}{ }_{N T}-\mathrm{mCD} 74-V 5$ & \begin{tabular}{|l|l|}
$\mathrm{HA}$ \\
\end{tabular} & LL & TM & CLiP & V5 \\
\hline & $\left.\right|^{2}$ & 52 & & & $38 \mid$ \\
\hline HA-TMEM192 ${ }_{\mathrm{NT}}-\mathrm{mCD} 74-\mathrm{V} 5$ & $\mathrm{HA}$ & LL LL & $\mathrm{TM}$ & CLiP & V5 \\
\hline
\end{tabular}

B

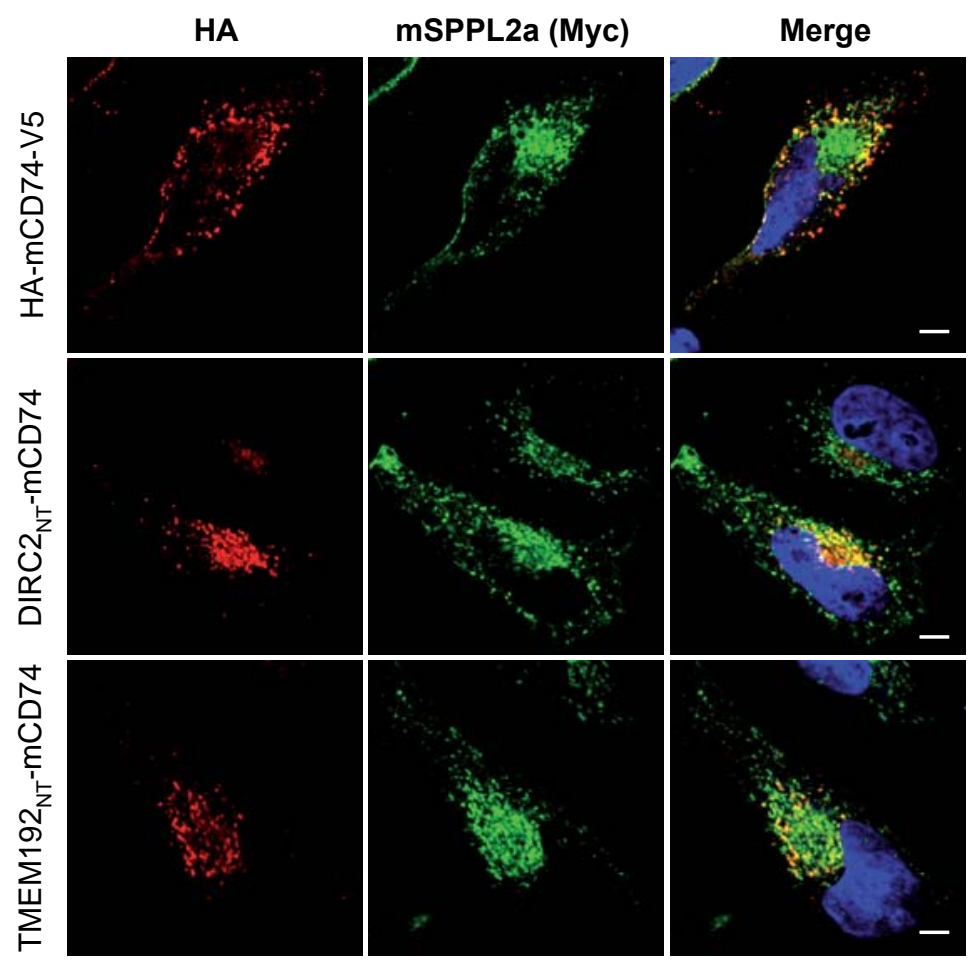

C

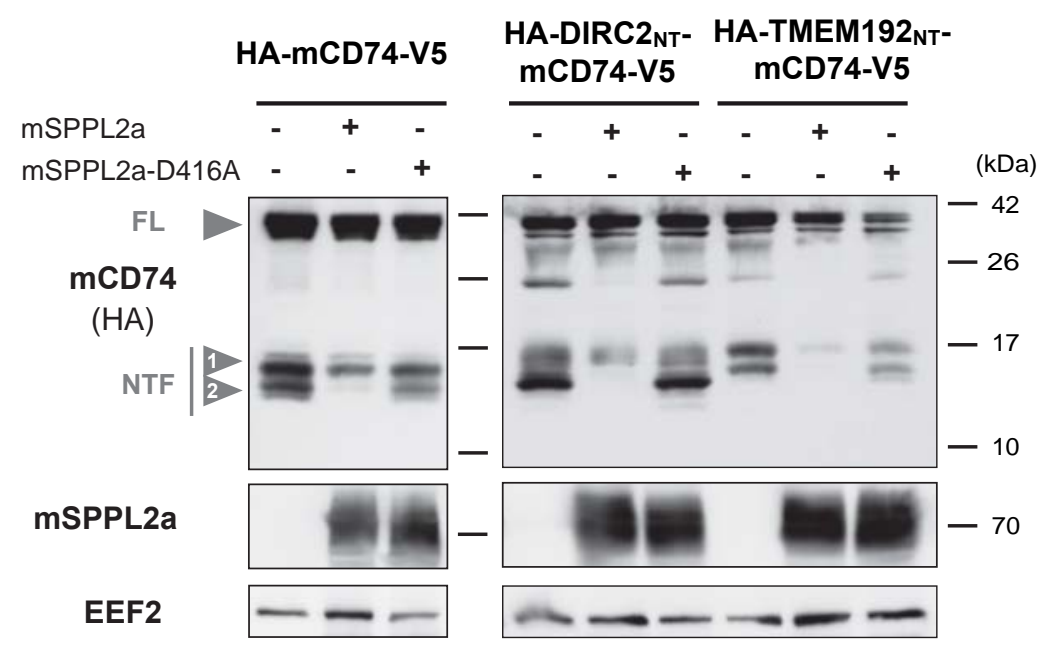


A

\begin{tabular}{|c|c|c|c|c|c|c|}
\hline \multirow{2}{*}{\multicolumn{2}{|c|}{ mCD74-HA }} & TM & & \multicolumn{2}{|l|}{ CLiP } & \multirow[t]{2}{*}{ HA } \\
\hline & & 1 & 455 & 83 & 215 & \\
\hline mCD74 p31-HA & \multicolumn{2}{|c|}{ MDDQRDLISNHEQLPILGNRPREPERCSR } & \multicolumn{2}{|c|}{ GALYTGVSVLVALLLAGQATTAYFL } & \\
\hline 18-23A-HA & \multicolumn{2}{|c|}{ MDDQRDLISNHEQLPILAAAAAAPERCSR } & \multicolumn{2}{|c|}{ 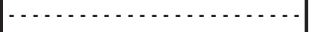 } & \multicolumn{2}{|c|}{ YQ. . . . . . . . . . . . . . . . . . . } \\
\hline 24-29A-HA & \multicolumn{2}{|c|}{ MDDQRDLISNHEQLPILGNRPREAAAAAA } & \multirow{2}{*}{\multicolumn{2}{|c|}{ AALYTGVSVLVALLLAGQATTAYFL }} & \multicolumn{2}{|c|}{ 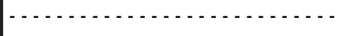 } \\
\hline 30A-HA & \multicolumn{2}{|c|}{ (n) } & & & \multicolumn{2}{|c|}{ 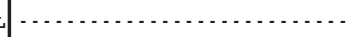 } \\
\hline 32-37A-HA & \multicolumn{2}{|c|}{ (n, } & \multicolumn{2}{|c|}{ GAAAAAAAVLVALLLAGQATTAYFL } & \multicolumn{2}{|c|}{ (n. } \\
\hline $38-40 \mathrm{~A}-\mathrm{HA}$ & & \multicolumn{2}{|c|}{ GALYTGVSAAAALLLAGQATTAYFL } & \multicolumn{2}{|c|}{$\mid \cdots, \ldots$} \\
\hline 42-44A-HA & & \multicolumn{2}{|c|}{ GALYTGVSVLVAAAAAGQATTAYFL } & \multicolumn{2}{|c|}{ 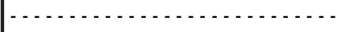 } \\
\hline 46-50A-HA & & \multicolumn{2}{|c|}{ GALYTGVSVLVALLLAAAAAAAYFL } & \multicolumn{2}{|c|}{ 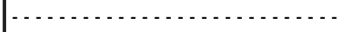 } \\
\hline 52-54A-HA & & \multicolumn{2}{|c|}{ GALYTGVSVLVALLLAGQATTAAAA } & \multicolumn{2}{|c|}{ 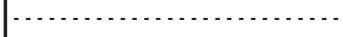 } \\
\hline 55-60A-HA & \multirow{2}{*}{\multicolumn{2}{|c|}{ 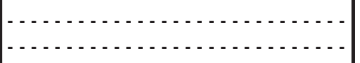 }} & $\cdots$ & ( & \multicolumn{2}{|c|}{ AAAAAALDKLTITSQNLQLESLRMKLPK } \\
\hline 61-66A-HA & & & & & \multicolumn{2}{|c|}{ YQQQGRAAAAAATSQNLQLESLRMKLPK } \\
\hline 67-72A-HA & \multicolumn{2}{|l|}{ (......... } & & & \multirow{2}{*}{\multicolumn{2}{|c|}{$\begin{array}{l}\text { YQQQGRLDKLTIAAAAAALESLRMKLPK } \\
\text { YQQQGRLDKLTITSQNLQAAAAAAKLPK }\end{array}$}} \\
\hline 73-78A-HA & & & & & \\
\hline 79-82A-HA & & & & \multicolumn{2}{|c|}{ YQQQGRLDKLTITSQNLQLESLRMAAAA } \\
\hline
\end{tabular}

B

mCD74-HA

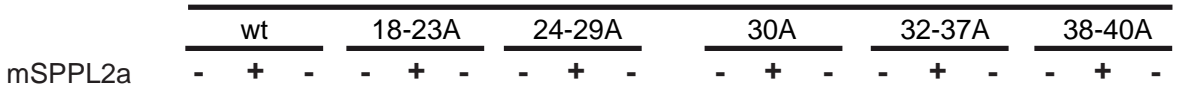

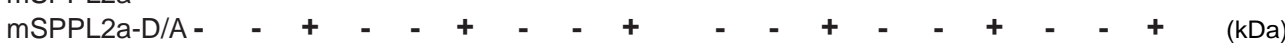

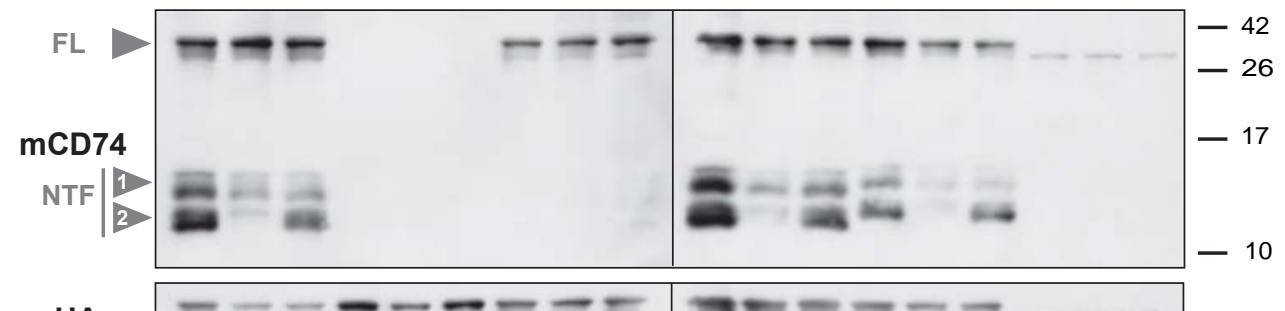

HA

m-

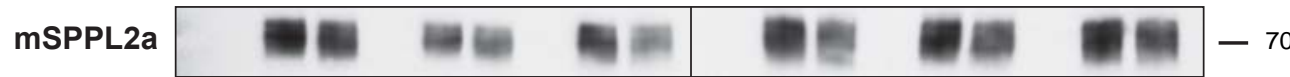

EEF2 - - - - - - - - - -

mCD74-HA

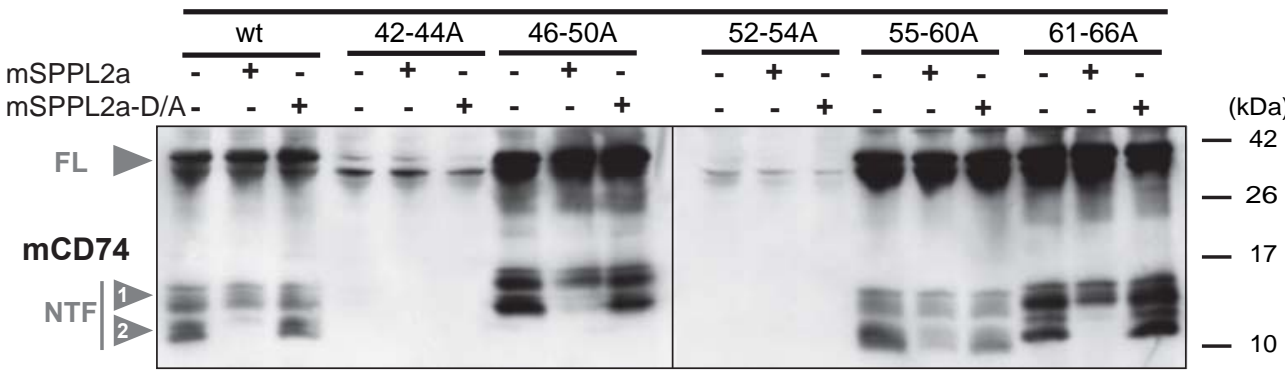

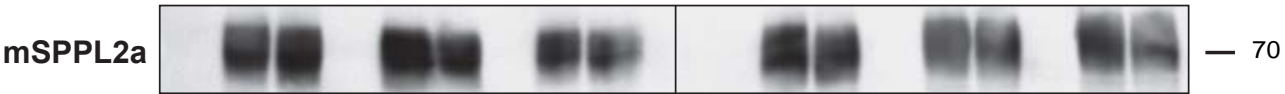

GAPDH

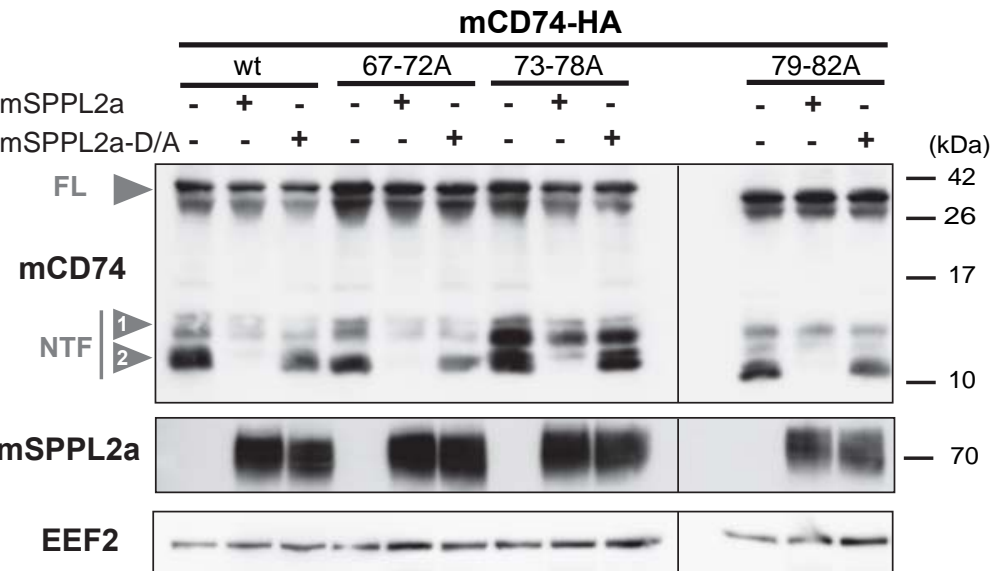

C

mSPPL2a $=+-\div+-$ $\mathrm{mSPPL} 2 \mathrm{a}-\mathrm{D} / \mathrm{A}-\quad+\quad+\quad+\quad+\quad \mathrm{kDa})$

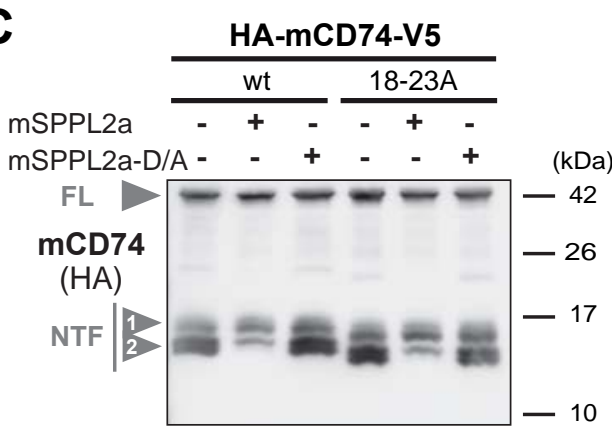

mSPPL2a

EEF2 $-\ldots \ldots$ 


\section{FIGURE 5}

A

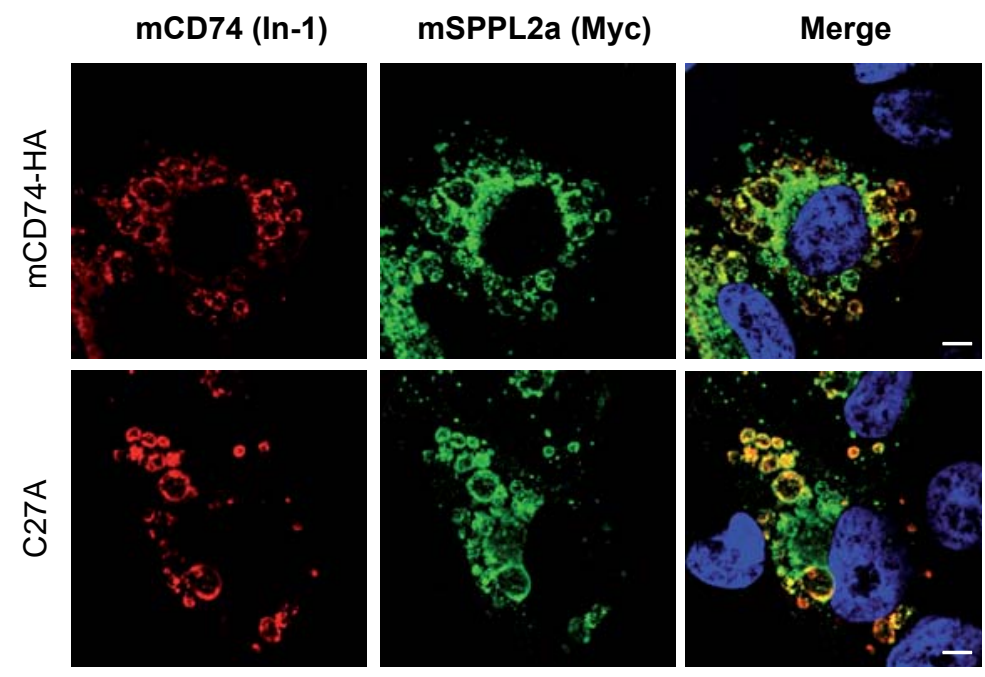

B

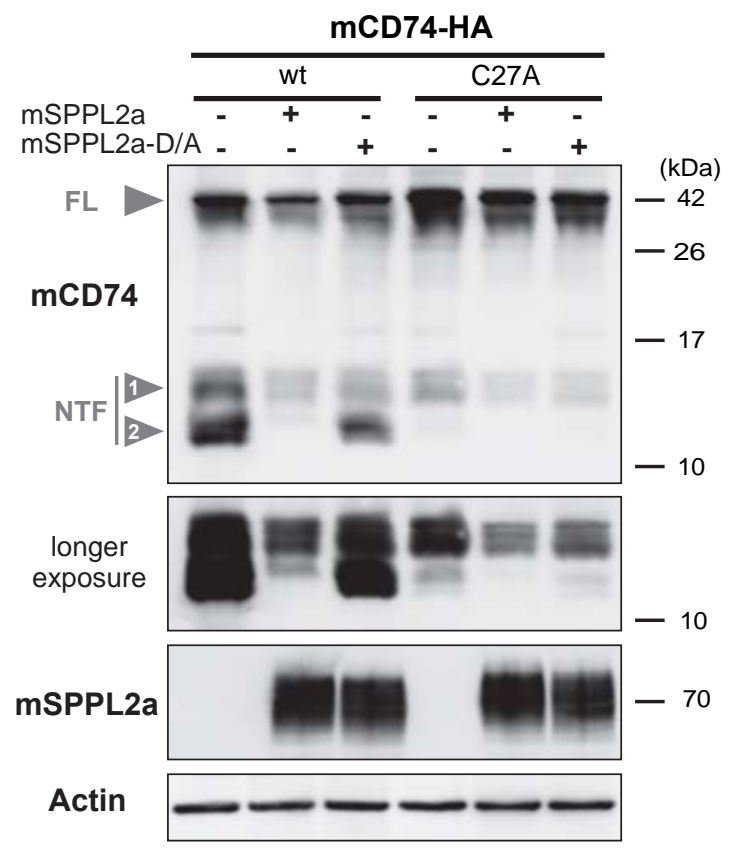


A

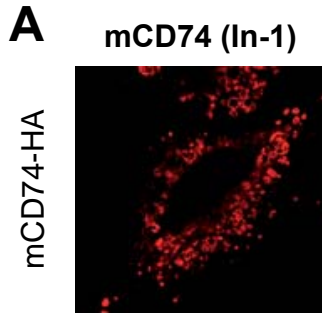

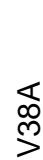

ঙ্ণ

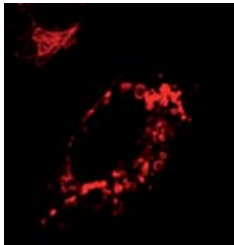

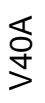
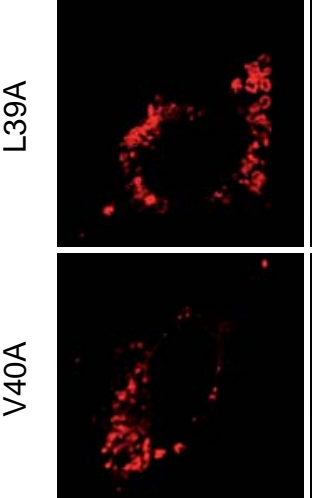

$\stackrel{\nwarrow}{\lessgtr}$

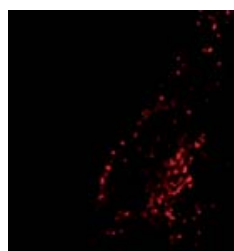

㜽

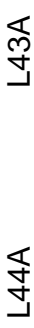
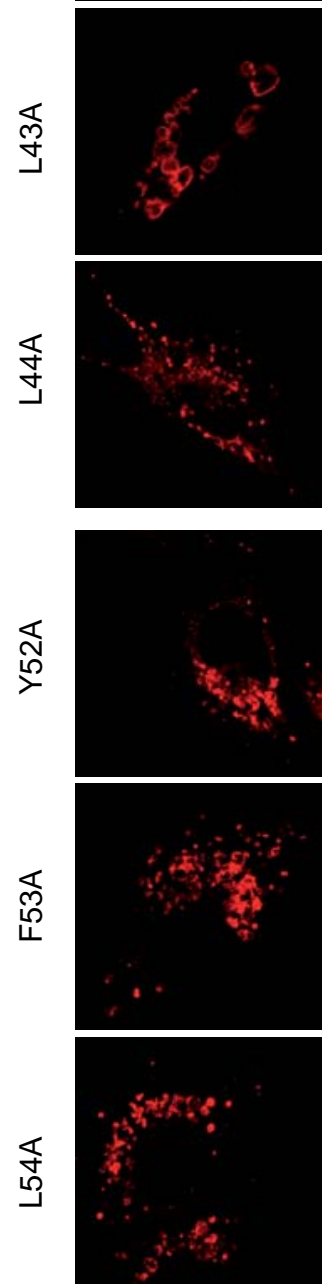

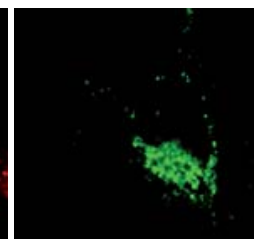

Merge
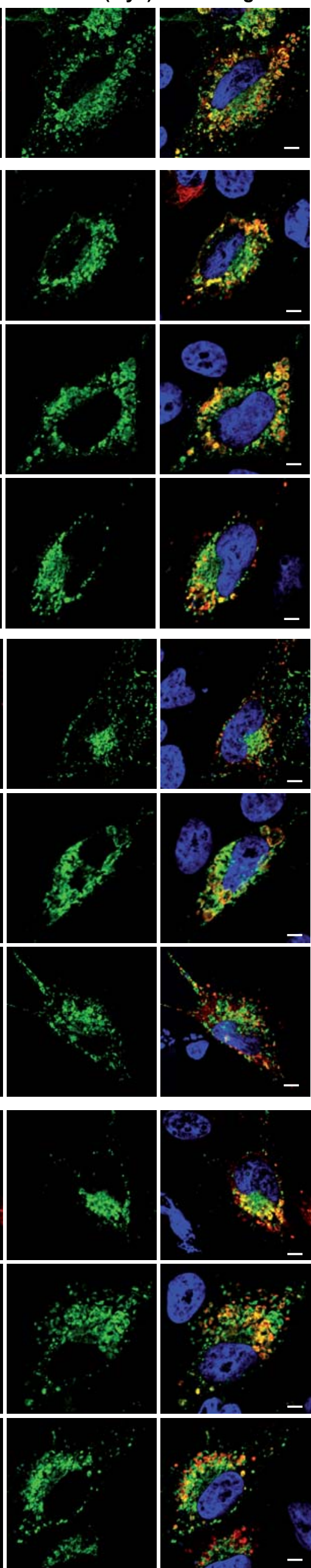

B

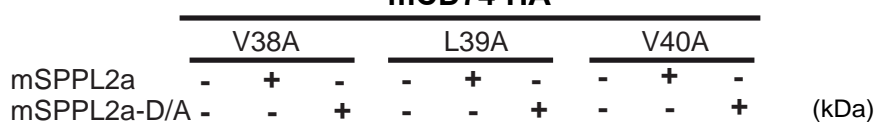

mSPPL2a-DIA

mCD74

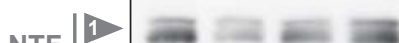

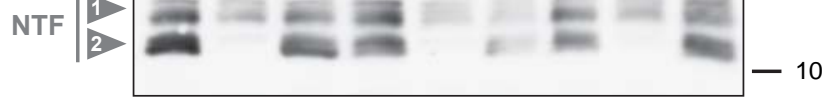

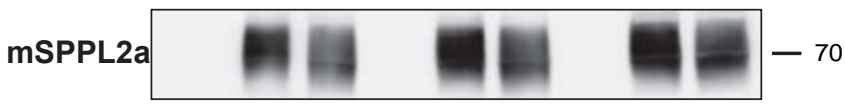

EEF2

C

\begin{tabular}{|c|c|c|}
\hline & CD74-HA & \\
\hline L42A & L43A & L44A \\
\hline
\end{tabular}

mSPPL2a

mSPPL2a-D/A - - + - + $-\quad+\quad+\quad(\mathrm{kDa})$

$\mathrm{FL} \Rightarrow-\mathrm{F}=\mathrm{F}=$

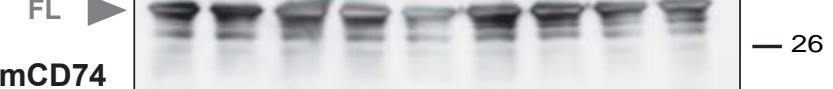

- 1 - $-2=-17$

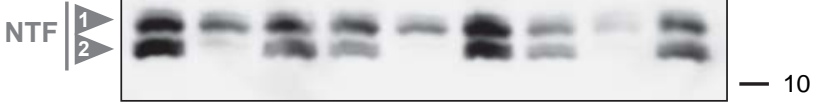

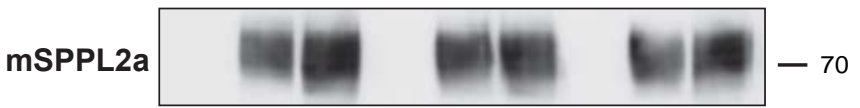

Actin $-\ldots \ldots$

D

$\frac{\text { CD74-HA }}{\frac{\text { Y52A }}{-+553 A}-\frac{\text { L54A }}{-+\frac{+}{-}-}}$

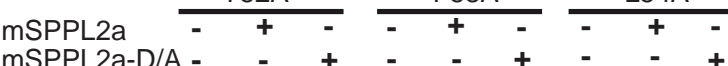

$-\quad+\quad(\mathrm{kDa})$

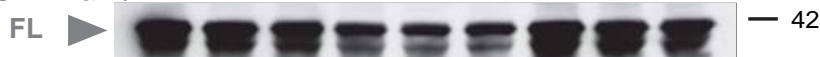

mCD74

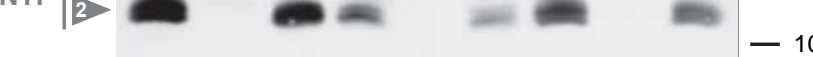

\begin{tabular}{c|c|c|} 
mSPPL2a & A \\
EEF2 & $-\infty-\infty$
\end{tabular} 


\section{FIGURE 7}

A

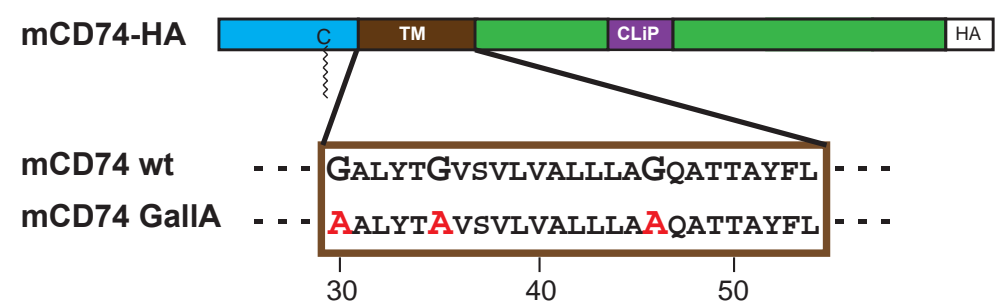

B

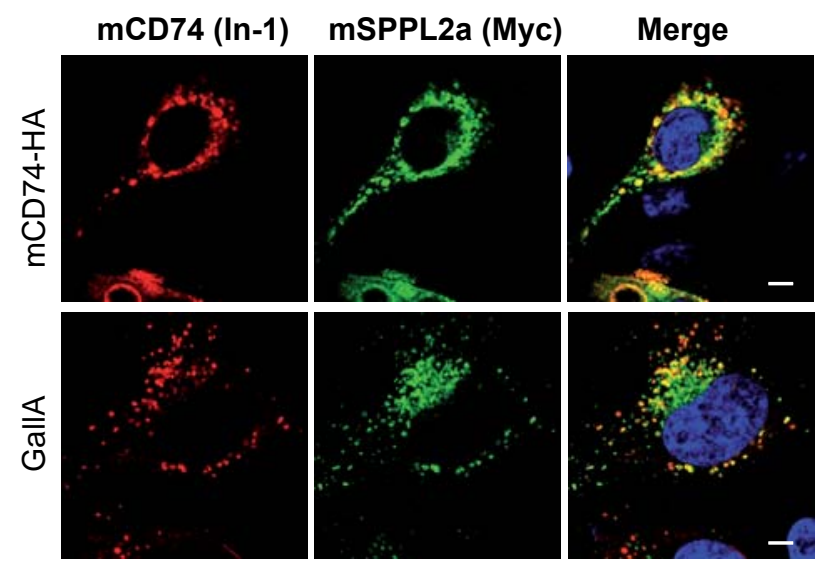

C

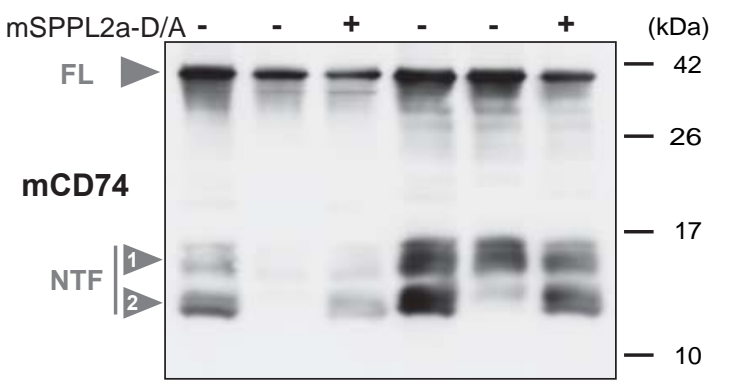

mSPPL2a

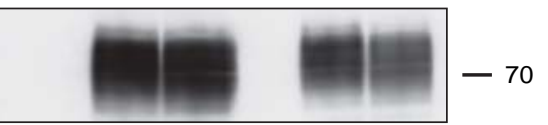

Actin

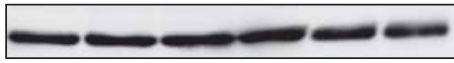

D

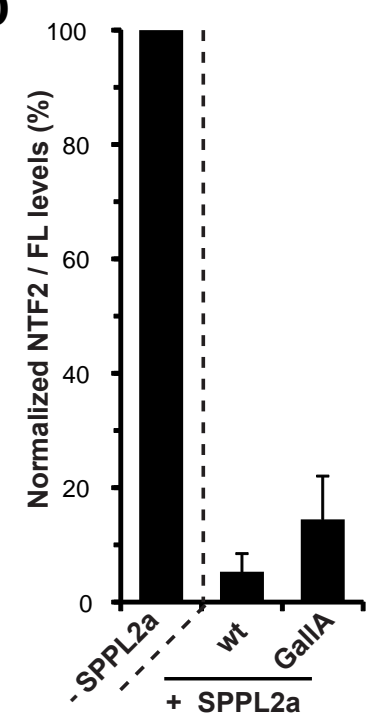

E

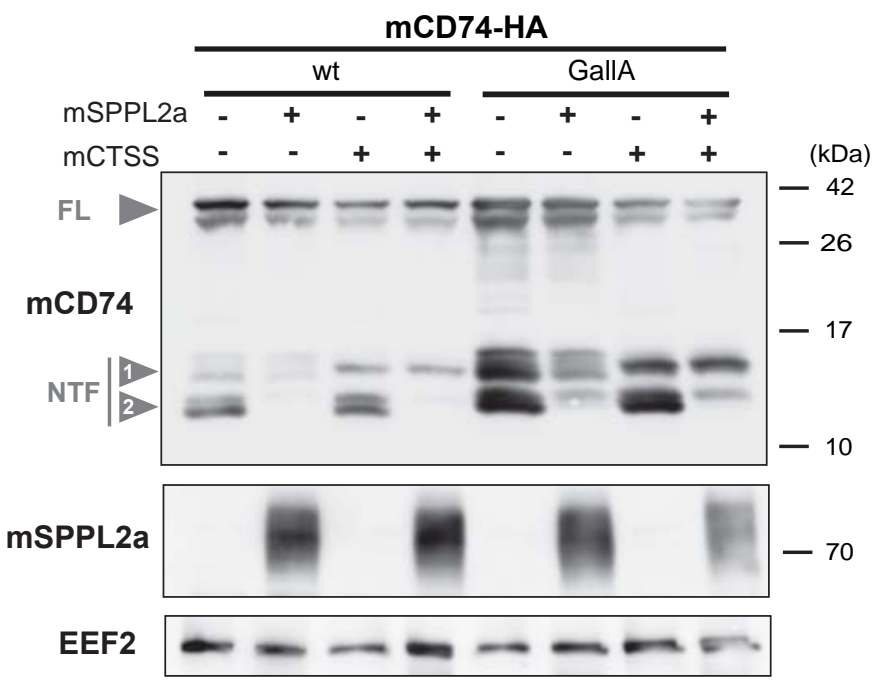


FIGURE 8

A

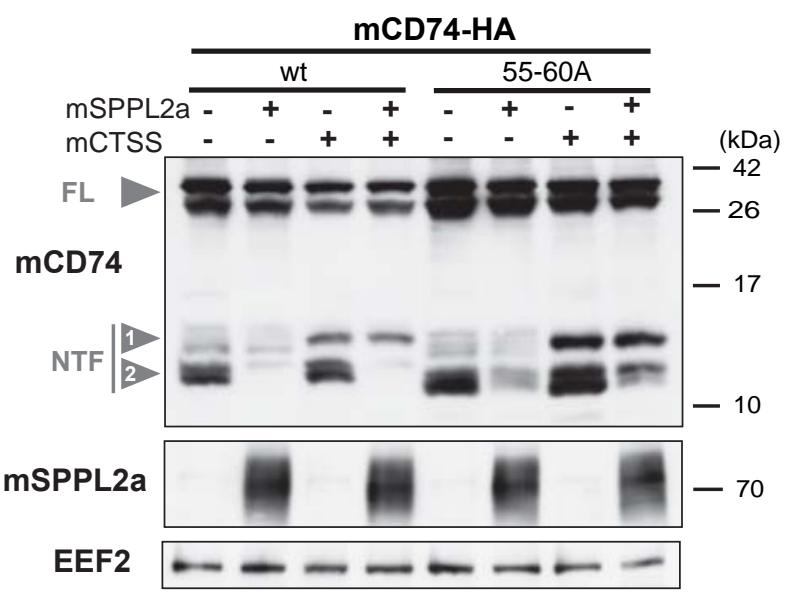

C

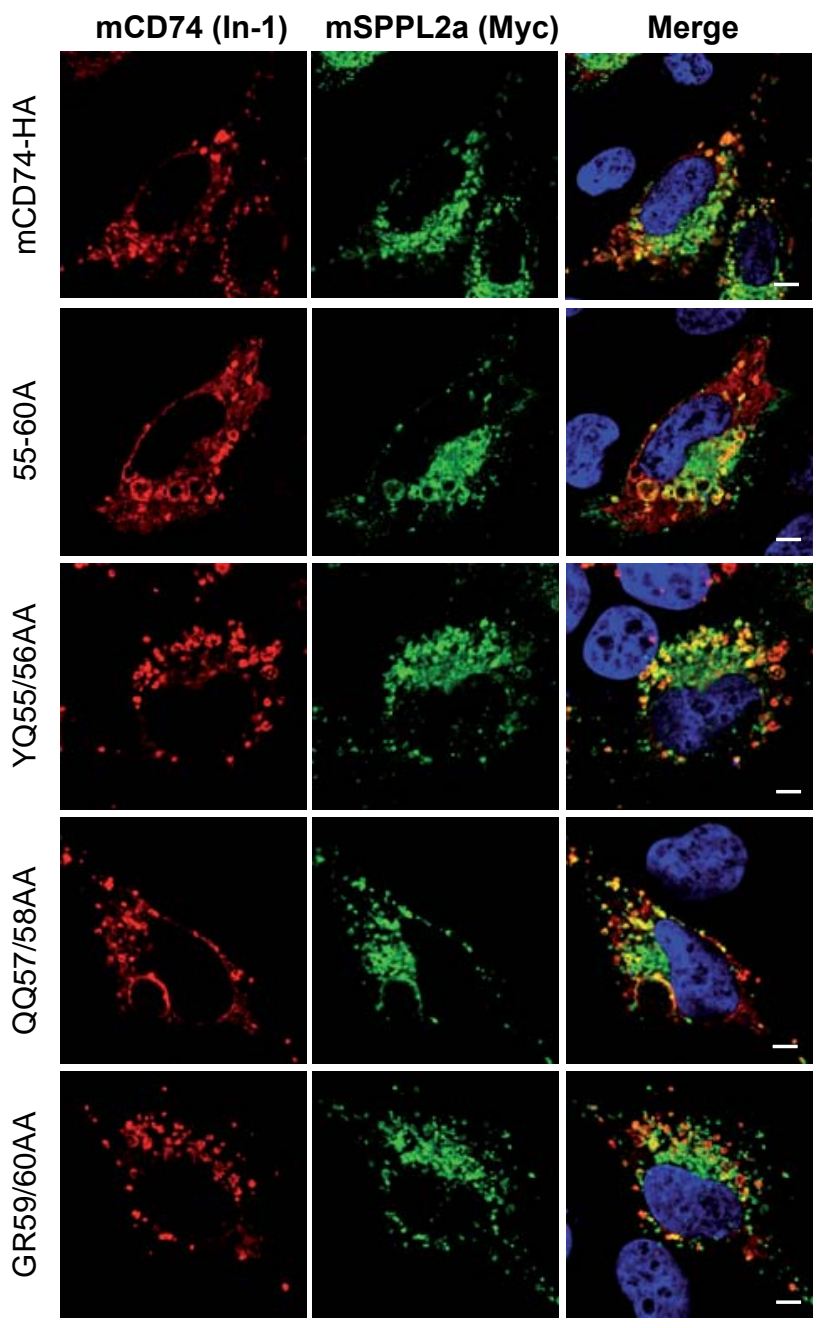

B

D
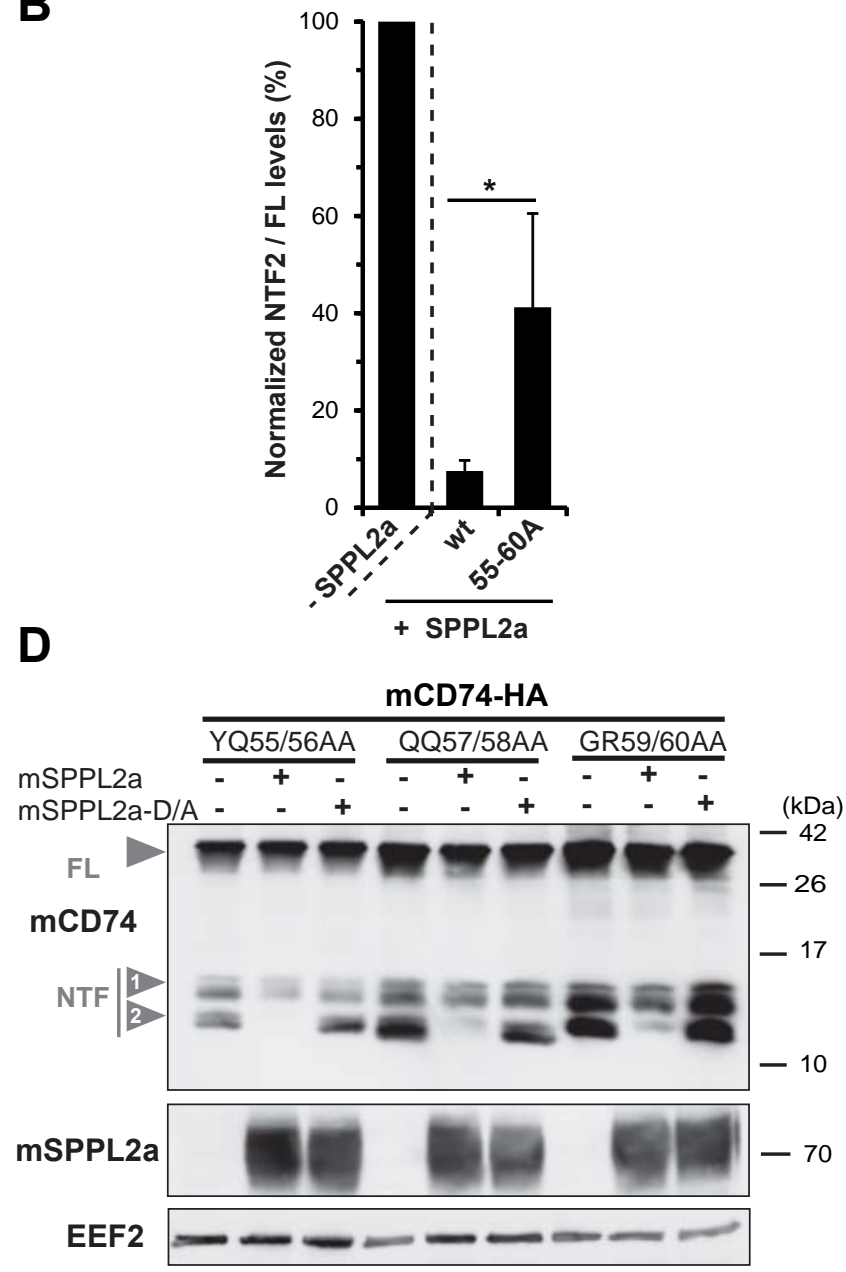

E

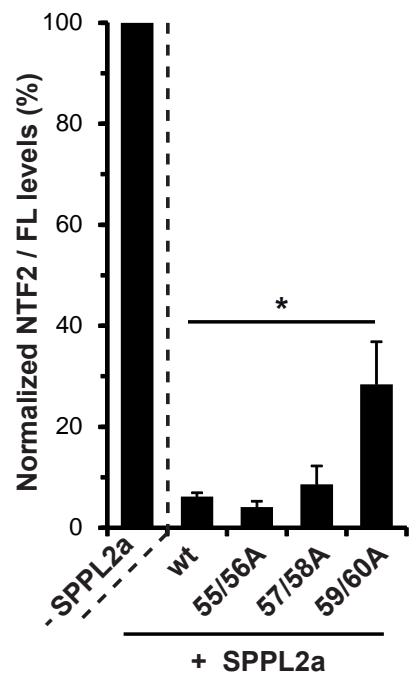

F mCD74-HA

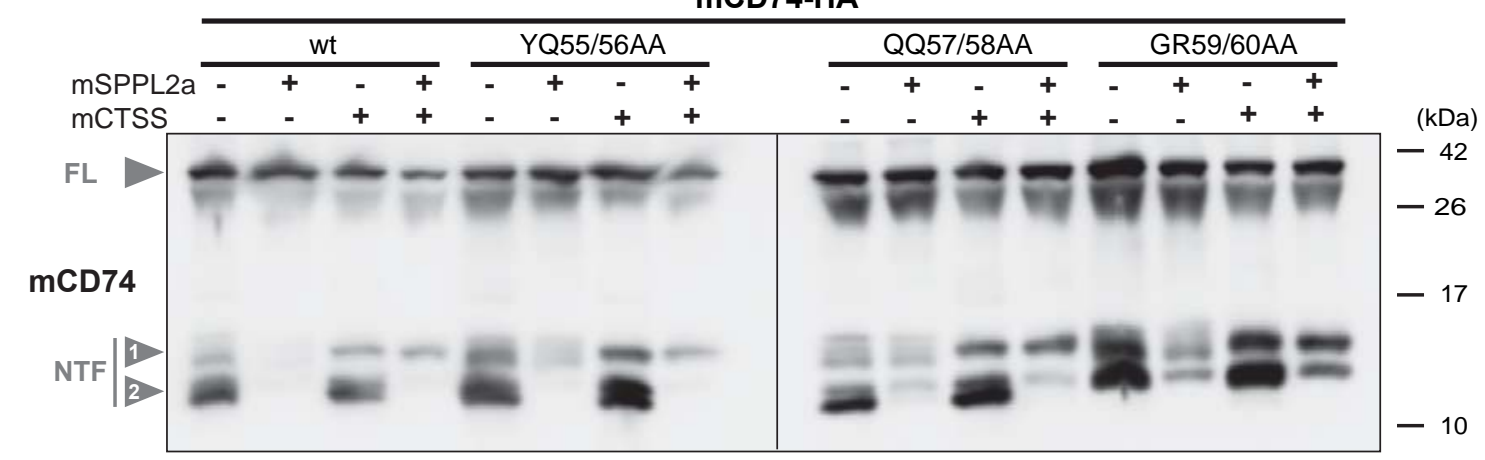

mSPPL2a 


\section{FIGURE 9}

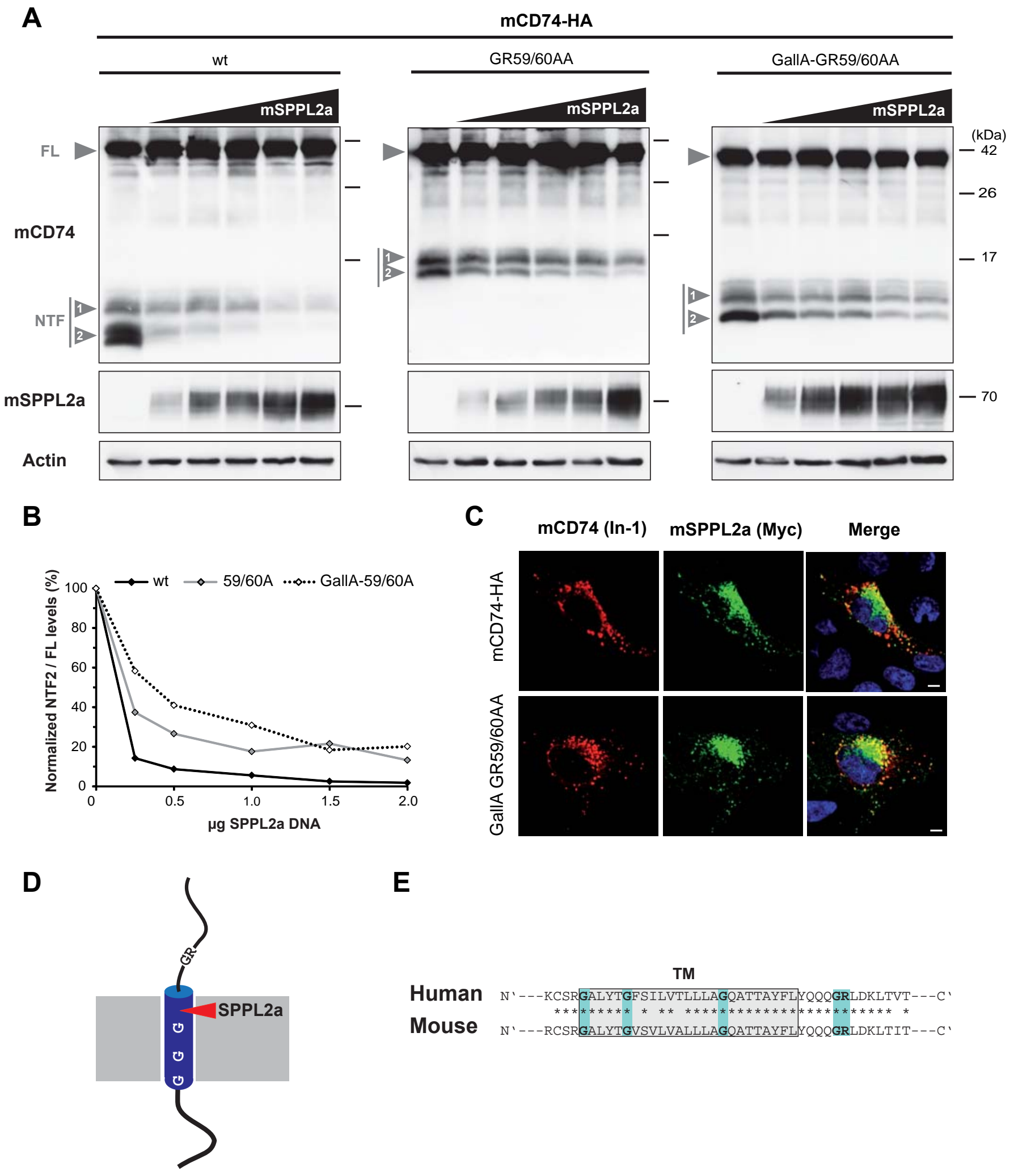

